Ann. Zootech., I966, 15 (I), I5-45.

\title{
RECHERCHES SUR LES POSSIBILITÉS D'EMPLOI DE LA BARYMÉTRIE CHEZ LES BOVINS
}

\author{
B. VISSAC \\ avec la collaboration technique de M. LE RoullLy \\ Station centrale de Génétique animale, \\ Centre national de Recherches zootechniques, 78-Jouy-en-Josas
}

\section{SOMMAIRE}

A l'occasion de la mise au point de formules de barymétrie sur des échantillons d'animaux de sexes, d'âges et d'états d'engraissement variés et appartenant à des races exploitées en France on a fait un certain nombre d'études sur les possibilités et conditions d'utilisation de cette méthode.

L'intérêt d'un emploi séparé ou combiné des 2 mesures les plus efficaces pour estimer le poids vif (tour de poitrine et tour spiral) est discuté. L'adjonction de la $2^{\mathbf{e}}$ mesure à la première dans une équation de régression linéaire est surtout intéressante pour des animaux adultes.

La précision des ajustements linéaires utilisant les puissances entières d'ordre I,2 ou 3 de ces 2 variables est étudiée. L'emploi des puisances supérieures à I n'est justifié que pour les échantillons de bovins en croissance : dans ce cas, le meilleur ajustement correspond en général à la puissance 2 du tour de poitrine et à la puissance 3 du tour spiral.

Les paramètres statistiques de ces ajustements varient suivant les échantillons avec le sexe, la race et l'âge moyen des animaux. L'incidence de l'état d'engraissement sur ces paramètres semble plus accusée avec le tour de poitrine qu'avec le tour spiral. toire.

La précision des 2 mesures envisagées est étudiée et analysée en fonction de la technique opéra-

L'intérêt respectif des estimations directe et indirecte du poids vif en vue du contrôle de descendance réalisé dans les fermes est enfin discuté : la $2^{\mathrm{e}}$ méthode est théoriquement préférable si le coût unitaire de son emploi est inférieur de ıo $p$. ıoo au coût de la pesée.

\section{I. - INTRODUCTION ET BUT DU TRAVAIL,}

Le contrôle du poids des animaux a pris, ces dernières années, une importance croissante dans le cadre des opérations de contrôle zootechnique des bovins. Chez le jeune animal, il constitue l'élément de base du contrôle de la vitesse de croissance indispensable tant pour la sélection que pour l'étude des plans d'alimentation ou des méthodes d'élevage. On désire, par ailleurs, connaître le format de la femelle adulte, dont dépendent ses besoins d'entretien, pour le rapporter à sa production de lait. 

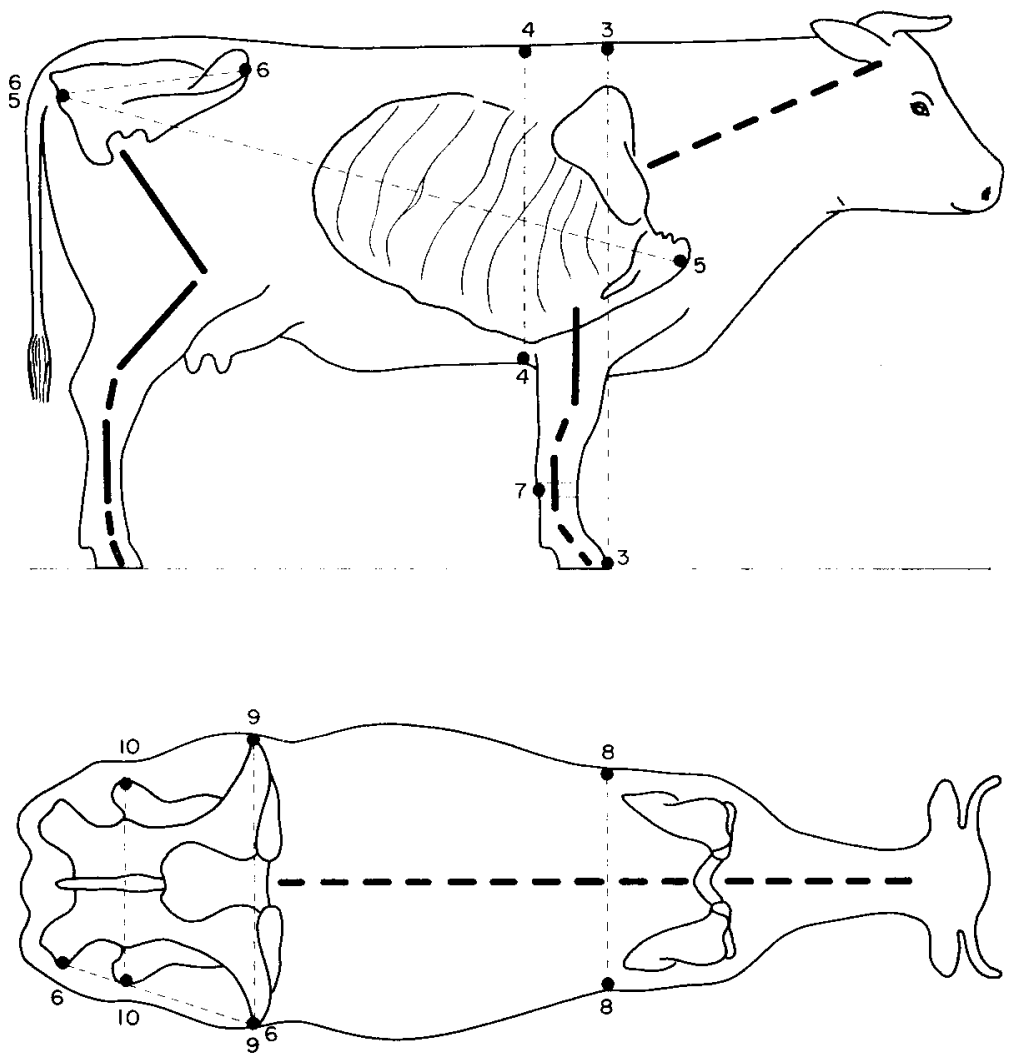

GRAPHIQUe I. - Mensuraiions de l'animal vivant

$\mathrm{X}_{3}$ : hauteur au garrot $\quad \mathrm{X}_{7}$ : tour de canon

$\mathrm{X}_{4}$ : hauteur de poitrine $\quad \mathrm{X}_{8}^{7}$ : largeur de poitrine

$\mathrm{X}_{5}$ : longueur totale $\quad \mathrm{X}_{9}$ : largeur aux hanches

$\mathrm{X}_{6}^{5}$ : longueur du bassin $\quad \mathrm{X}_{10}^{9}:$ largeur aux trochanters.

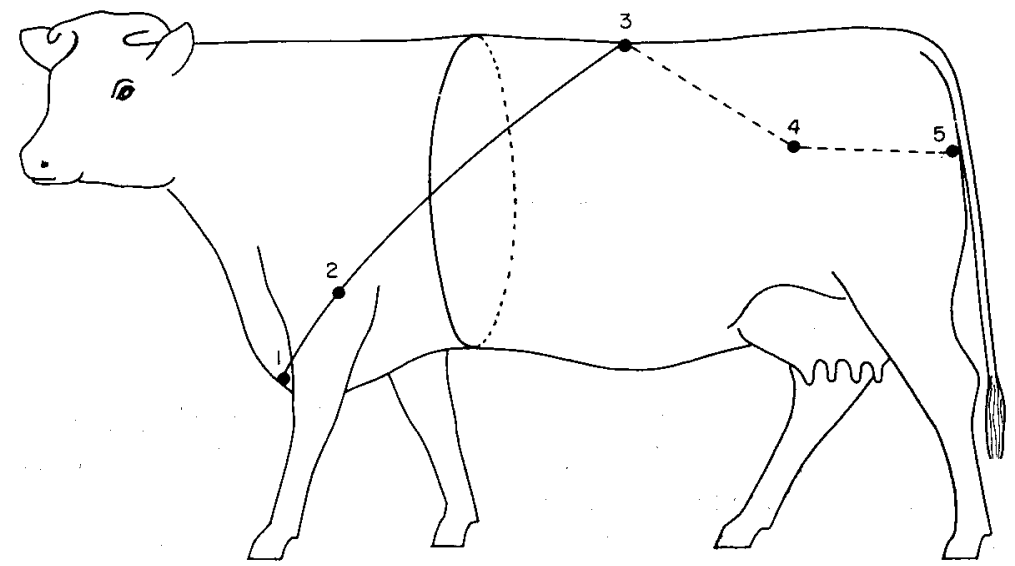

GRAPHiQue 2. - Tour de poitrine $\left(X_{1}\right)$. Tour spiral $\left(X_{2}\right)$.

I. pointe du sternum 4. une main sous la hanche.

2. milieu du bras

5. périnée

3. limite dos-rein 
La pesée du bovin, au-delà de $200 \mathrm{~kg}$ tout au moins, nécessitant l'emploi d'une bascule, le coût de l'opération s'avère bien souvent plus élevé que le bénéfice que l'on peut attendre de la connaissance de ce poids. Par ailleurs, l'objectif zootechnique étant souvent la déternination de poids moyens, de croissances moyennes de lots d'animaux issus de différentes fermes, de différents taureaux ou soumis à des régimes divers, l'erreur sur la moyenne dépend plus des fluctuations d'échantillonnage du groupe considéré que des variations aléatoires du résultat d'une pesée ; on peut alors être conduit à préférer une méthode grossière utilisable sur un effectif important plutôt qu'une technique très précise réalisable, pour le même coût, sur un nombre d'animaux restreint. Ces deux considérations peuvent expliquer l'intérêt d'une estimation indirecte du poids par des mesures corporelles simples.

Dans une étude préliminaire (1)ELAGE et $a l$., I955) une revue bibliographique des recherches consacrées à cette méthode a été effectuée. Les premières recherches personnelles que nous avons conduites nous ont ensuite amené à proposer une méthode d'estimation du poids vif des femelles adultes et de jeunes bovins de race Normande par barymétrie (I,E RouliLY et al., I958; VISSAC, I959). Cette méthode a été étendue et appliquée aux bovins des différentes races françaises en liaison avec l'Union nationale des I,ivres généalogiques (NuUvy, I962).

Le but de ce mémoire est de rassembler les résultats de recherches effectuées par nous-même, en France, de 1955 à I962, pour justifier la méthode proposée d'un part, pour en définir les conditions et limites d'application d'autre part.

\section{II. - MÁTÉRIEI, D'ÉTUDE:}

Le matériel aninal utilisé pour ces études était constitué d'échantillons de bovins de races, de sexes, d'âges et d'états d'engraissement variés et contrôlés soit à l'occasion du Concours général agricole (1952-1955), soit dans des abattoirs : Ia Villette, Lisieux (1954-1958), ou dans des fermes à l'occasion d'opérations de contrôle d'aptitude $\left(1959^{-1}\right.$ I $^{6} 3$ ) : contrôle laitier ou contrôle de croissance. Les caractéristiques de ces échantillons que nous désignerons par $\mathfrak{E}_{\text {; }}$ sont rassemblées sur le tablean $\mathbf{r}$. Une partie de ce matériel d'étude a été utilisée ì l'occasion de premières recherches effectuées sur ce sujet : les échantillons correspondants et les recherches auxquelles ils ont donné licu sont précisés également sur le tableau 1.

Les poids de carcasse $\left(\mathrm{Y}^{\prime}\right)$ ont été obtenus sur des carcasses froides et dégraissées; les poids vifs (Y) correspondent a des pesées effectuées à des heure's variables de la journée et sans tenir compte de l'heure des repas. Les mensurations envisagées sont représentées schématiquement sur les graphiques 1 et 2 ; nous utiliserons pour les désigner dans la suite de ce texte les abréviations $\left(\mathrm{X}_{i}\right)$ figurant au bas de ces graphiques. Elles étaient réalisées par un seul opérateur et une fois par animal ; la longueur totale $\left(\mathrm{X}_{2}\right)$ et le tour spiral $\left(\mathrm{X}_{3}\right)$ étant sistématiquement répétés.

\section{III. 一 RÉSULTATS STATISTIQUES}

La recherche d'une estimation statistique du poids par des mensurations comporte plusieurs étapes que nous aborderons successivenent :

- le choix des mesures à retenir pour cette estimation;

- le choix du type d'ajustement statistique le plus précis ;

- l'étude des facteurs de variation de l'équation d'estimation ;

- la comparaison des avantages théoriques et économiques de cette méthode par rapport à la pesée.

Annales de Zootechnie. - igr:6. 


\begin{tabular}{|c|c|c|c|c|c|c|c|}
\hline 总 & 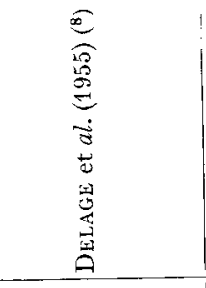 & 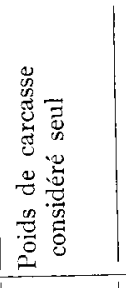 & & 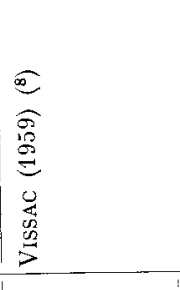 & 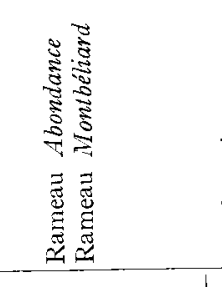 & 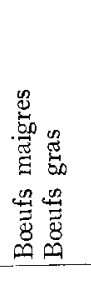 & \multirow{5}{*}{ 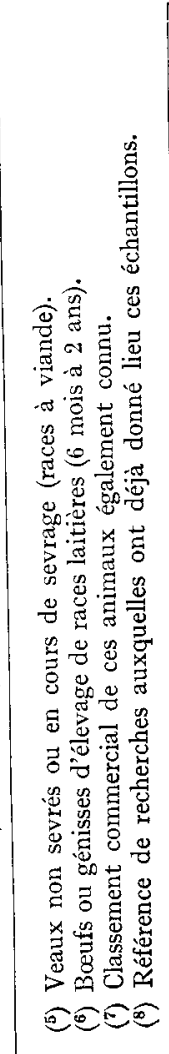 } \\
\hline 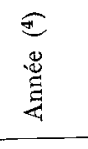 & 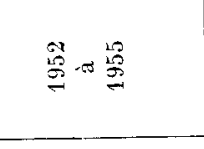 & 营莺点 & 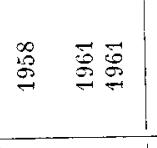 & 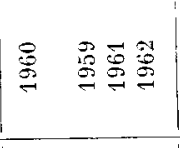 & | & 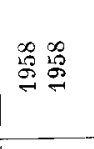 & \\
\hline 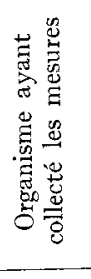 & 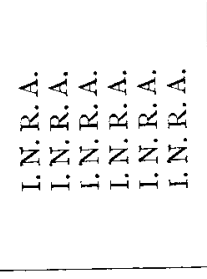 & 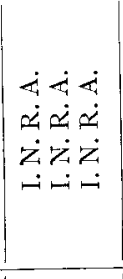 & 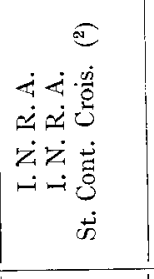 & 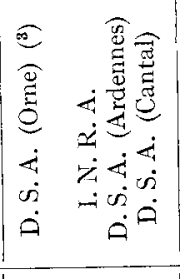 & 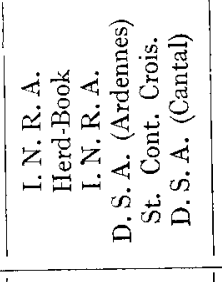 & 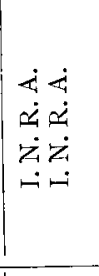 & \\
\hline $\begin{array}{l}\text { 岕 } \\
\text { 离 } \\
\text { 四 }\end{array}$ & 영영 & 路起星 & 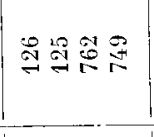 & 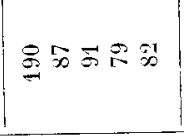 & 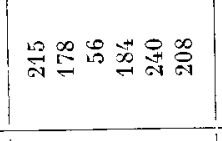 & $\stackrel{0}{*} \stackrel{0}{=}$ & \\
\hline$\underset{\mathscr{\varpi}}{\mathscr{u}}$ & 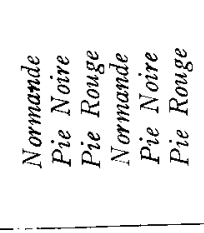 & 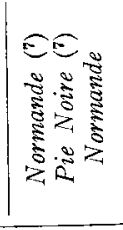 & 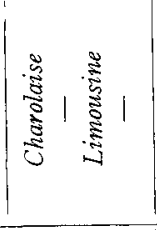 & 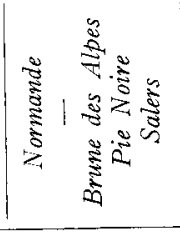 & 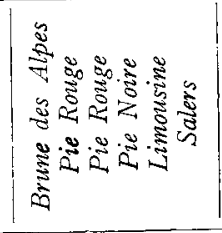 & 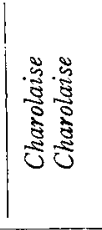 & \\
\hline 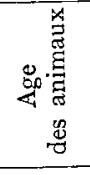 & 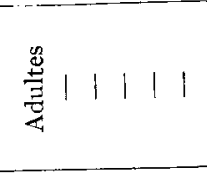 & 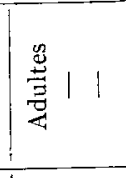 & ${ }_{\substack{3 \\
0}}^{a} 11$ & 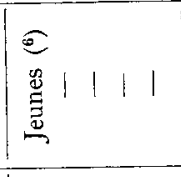 & $\frac{0}{\frac{3}{3}}|1| 1 \mid$ & 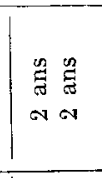 & $\begin{array}{l}\dot{\dot{\alpha}} \\
\dot{x} \\
\dot{z} \\
\dot{b}\end{array}$ \\
\hline 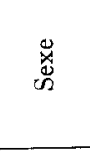 & 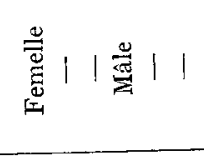 & 咅 11 & 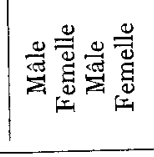 & 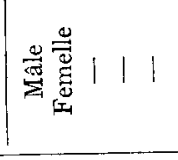 & 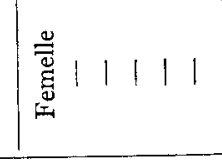 & 袮 & 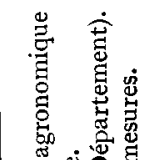 \\
\hline 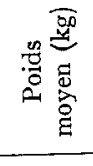 & 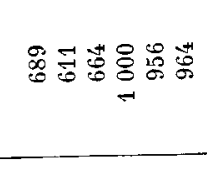 & 卷突芯 & 范茟芯合 & 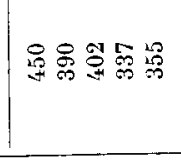 & 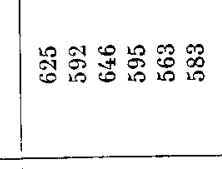 & 曽含 & 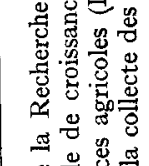 \\
\hline 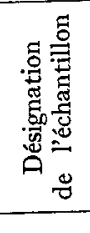 & 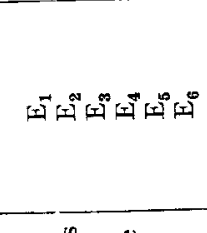 & Gif & 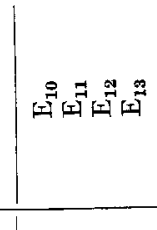 & 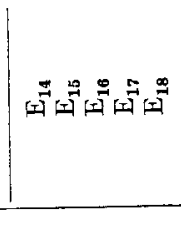 & 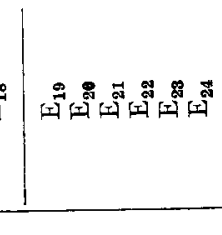 & 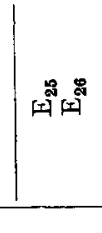 & 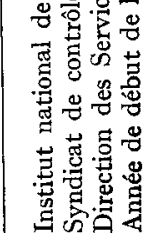 \\
\hline & 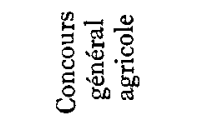 & 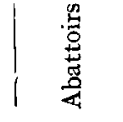 & I & & 离 & & こニの \\
\hline
\end{tabular}


Ces diverses études ont, pour des raisons d'ordre pratique ou liées à la nature du problème, porté sur tout ou partie des échantillons ci-dessus. Nous les présenterons néanmoins dans un ordre logique et d'une façon analytique.

\section{ChorX des MESURES}

\section{Ėtude préliminaire}

Une étude préliminaire a été effectuée en $\mathrm{I}_{95} 8$ sur 1'échantillon $\mathrm{E}_{9}=$ vaches Normandes de réforme.

Les coefficients de corrélation simple entre le poids vif (Y) ou le poids de carcasse $\left(\mathrm{Y}^{\prime}\right)$ et chacune des mensurations $\left(\mathrm{X}_{i}^{\prime}\right) r_{\mathrm{Y}}, \mathrm{x}_{i}, r_{\mathrm{Y}^{\prime}}, \mathrm{x}_{i}$ font apparaître l'intérêt $\mathrm{du}$ tour de poitrine $\left(\mathrm{X}_{1}\right)$ et du tour spiral $\left(\mathrm{X}_{2}\right)$ en vue de l'estimation du poids par une équation linéaire :

$$
\mathrm{Y} \text { ou } \widehat{\mathrm{Y}}^{\prime}=f\left(\mathrm{X}_{i}\right)(\text { tabl. } 2)
$$

Lorsque ces 2 variables sont associées dans une équation de régression linéaire multiple de la forme :

$$
\widehat{\mathrm{Y}} \text { out } \widehat{\mathrm{Y}^{\prime}}=f\left(\mathrm{X}_{1}, \mathrm{X}_{2}\right)
$$

les coefficients de corrélation multiple sont respectivement de 0,805 pour $\mathrm{Y}$ contre $0,75^{6}$ et $0,72 \mathrm{I}$ pour les coefficients de corrélation simples $r_{\mathrm{Y}}, \mathrm{x}_{1}, r_{\mathrm{Y}}, \mathrm{x}_{2}$, de 0,846 pour $\mathrm{Y}^{\prime}$ contre 0,802 et 0,745 pour $\gamma_{Y^{\prime}}, x_{1}$ et $r_{Y^{\prime}}, x_{2}$. Les écarts-types des distributions de $(\mathrm{Y}-\widehat{\mathrm{Y}})$ et $\left(\mathrm{Y}^{\prime}-\widehat{\mathrm{Y}^{\prime}}\right)$, dans le cas de $\mathrm{l}^{\prime}$ équation (2), sont respectivement de $24,68 \mathrm{~kg}$ et de $12,57 \mathrm{~kg}$.

L'adjonction d'une $3^{\mathrm{e}}$ variable aux deux précédentes dans une équation de régression linéaire :

$$
\widehat{\mathrm{Y}} \text { ou } \widehat{\mathrm{Y}}^{\prime}=f\left(\mathrm{X}_{1}, \mathrm{X}_{2}, \mathrm{X}_{i}\right)
$$

n'améliore pas sensiblement la valeur de la prédiction (tabl. 2); les coefficients de

TABIEAU 2

Précision des ajustements linéaires à 1 variable $\left(X_{\mathbf{i}}\right)$ ou à 3 variables $\left(X_{1}, X_{2}, X_{1}\right)$

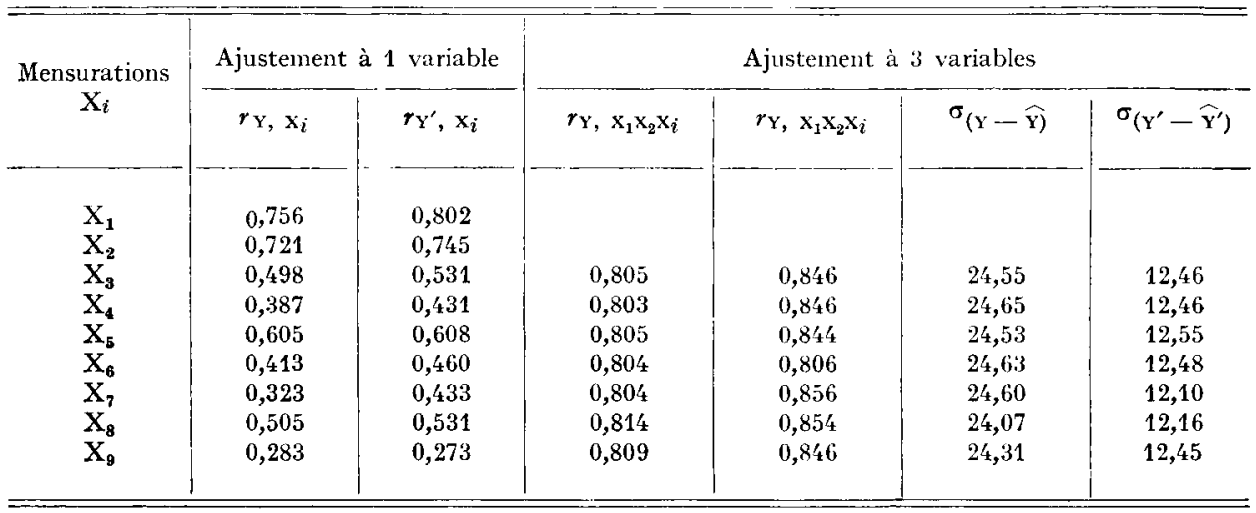


corrélation multiple $r_{\mathrm{Y}}, \mathrm{x}_{\mathrm{1}} \mathrm{x}_{2} \mathrm{x}_{i}$ et $\gamma_{\mathrm{Y}^{\prime}}, \mathrm{x}_{1} \mathrm{x}_{2} \mathrm{x}_{i}$ et les écarts-types des distributions résiduelles sont très voisins de ceux obtenus ci-dessus avec $X_{1}$ et $X_{2}$ seuls.

Il en est de même lorsque l'on s'intéresse à l'équation linéaire englobant les 9 variables considérées :

$$
\widehat{\mathrm{Y}} \text { ou } \mathrm{Y}^{\prime}=f\left(\mathrm{X}_{1} \cdots \mathrm{X}_{i}\right)
$$

Les coefficients de régression obtenus $\left(a_{i}\right)$ (tabl. 3) ne sont significativement différents de o (au seuil de $I$ p. Ioo) que pour $X_{1}$ et $X_{2}$. Les valeurs obtenues sont seulement à la limite du seuil de signification pour $\mathrm{X}_{7}$ et $\mathrm{X}_{8}$.

\section{TABIEAU 3}

Ajustement linéaire à 9 variables.

$$
\begin{aligned}
\widehat{\mathrm{Y}} & =\sum a_{i} \mathrm{X}_{i}+b, \\
\widehat{\mathrm{Y}}^{\prime} & =\sum{a^{\prime}}_{i} \mathrm{X}_{i}+b^{\prime} .
\end{aligned}
$$

\begin{tabular}{|c|c|c|c|c|}
\hline \multirow{2}{*}{$\mathrm{X}_{i}$} & \multicolumn{2}{|c|}{$Y$} & \multicolumn{2}{|c|}{$Y^{\prime \prime}$} \\
\hline & $a_{i}$ & $t\left(a_{i}\right)$ & $a_{i}^{\prime}$ & $f\left(a^{\prime} i\right)$ \\
\hline $\mathrm{X}_{1}$ & 2,29 & $5,70^{++}$ & 1,16 & $7,30++$ \\
\hline $\mathrm{X}_{2}$ & 1,96 & $3.30+1+$ & 0,96 & $3,30++$ \\
\hline$X_{3}$ & 0,08 & 0,13 & 0,15 & 0,48 \\
\hline$X_{4}$ & 11,89 & 1,000 & 0,80 & 1,82 \\
\hline $\mathrm{X}_{5}$ & 1,117 & 0,81 & 0,11 & 0,17 \\
\hline$X_{6}$ & 0,33 & 0,38 & 0,17 & 0,30 \\
\hline $\mathrm{X}_{7}$ & 14,59 & 1,19 & 1,56 & $3,08++$ \\
\hline $\mathrm{X}_{8}$ & 2,20 & $1,68+$ & 1.31 & $2,11+$ \\
\hline $\mathrm{X}_{9}$ & 0,32 & 1,72 & 0,11 & 1,07 \\
\hline
\end{tabular}

\section{Étude globale}

Pour les échantillons d'aninuaux suivis de I959 à I964 nous n'avons contrôlé, à la suite de cette première étude, que le tour de poitrine $\left(\mathrm{X}_{1}\right)$ et le tour spiral $\left(\mathrm{X}_{2}\right)$. Nous avons cherché à établir l'intérêt respectif de ces mesures dans l'estimation du poids. Les écarts-types des distributions résiduelles sont rassemblés dans le tableau 4 pour l'ensemble de ces échantillons et avec :

$$
\begin{aligned}
& \widehat{\mathrm{Y}}_{1}=f\left(\mathrm{X}_{1}\right)\left(\sigma_{1}\right) ; \\
& \widehat{\mathrm{Y}}_{2}=f\left(\mathrm{X}_{2}\right)\left(\sigma_{2}\right) ; \\
& \widehat{\mathrm{Y}_{3}}=f\left(\mathrm{X}_{1}, \mathrm{X}_{2}\right)\left(\sigma_{3}\right) .
\end{aligned}
$$

Nous étudierons successivement :

- l'intérêt respectif des estimations à partir du tour de poitrine et du tour spiral considérés isolément $\left(\widehat{\mathrm{Y}}_{1}\right.$ et $\left.\widehat{\mathrm{Y}}_{2}\right)$; 
- l'intérêt d'une estimation à partir de ces 2 variables $\left(\mathrm{X}_{1}, \mathrm{X}_{2}\right)$ par rapport à celle fournie par $\mathrm{X}_{1}$ seul, la mesure de $\mathrm{X}_{2}$ (tour spiral) étant délicate à réaliser $\left(\widehat{Y}_{1}\right.$ et $\left.\widehat{Y}_{3}\right)$

\section{TABLEAU 4}

Comparaison des erreurs d'ajustement $(\mathrm{kg})$ des équations de régression

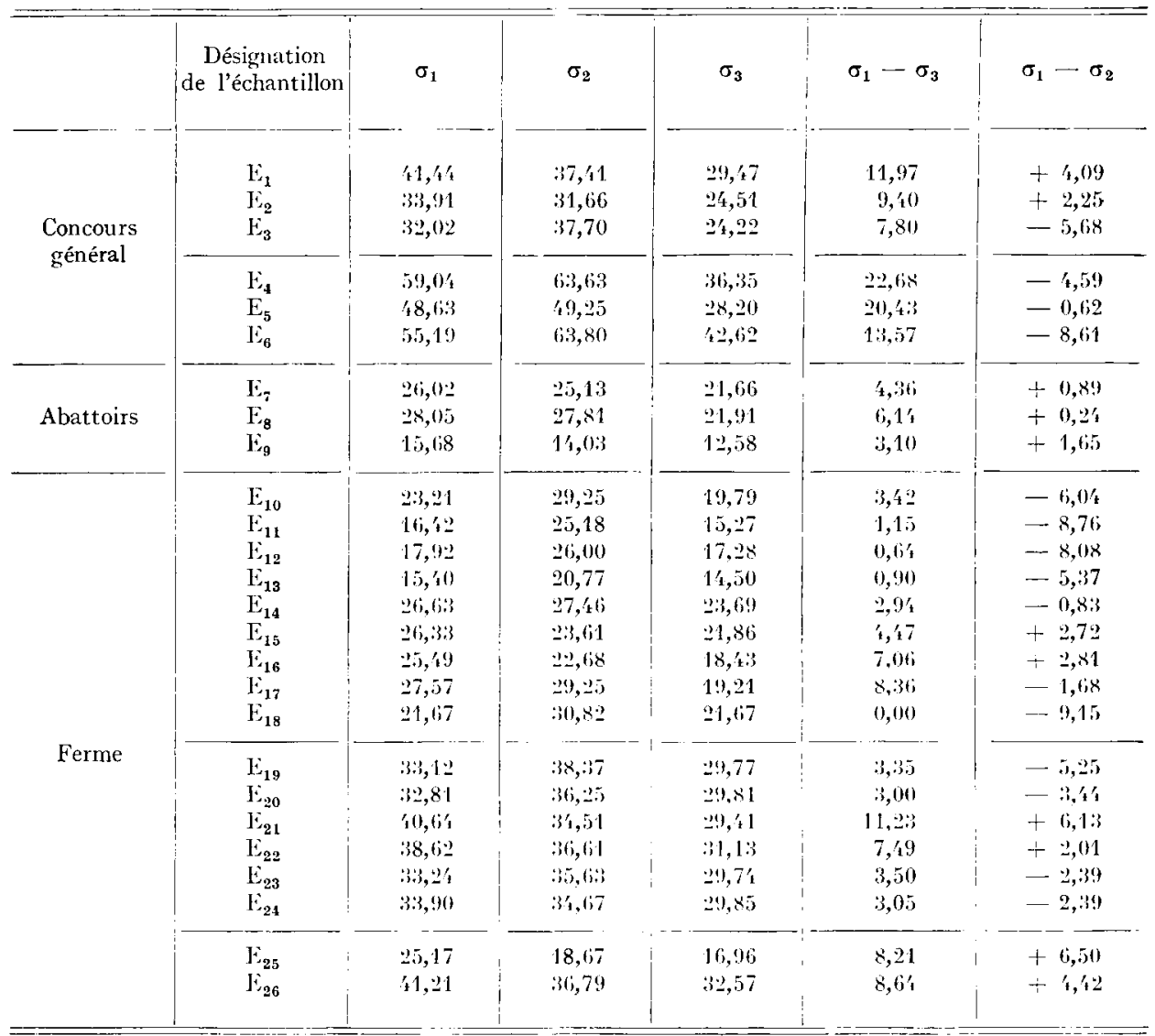

Comparaison des estimations $\widehat{Y}_{1}$ et $\widehat{Y}_{2}$.

Dans le cas des veaux de moins d'un an en races de boucherie, le tour de poitrine fournit une estimation meilleure que le tour spiral : la variance résiduelle est de 5 à 8 p. Ioo plus faible. Pour les échantillons d'animaux dont l'âge varie entre 6 mois et 2-3 ans, l'intérêt du tour spiral est plus grand : on remarque même une certaine liaison entre l'âge moyen des échantillons d'animaux en croissance et la différence $\sigma_{1}-\sigma_{2}$.

En ce qui concerne les animaux adultes, les résultats sont plus variables : le tour de poitrine paraît plus intéressant pour les échantillons de taureaux adultes 
$\left(\mathrm{E}_{4}, \mathrm{E}_{5}, \mathrm{E}_{6}\right)$ et le tour spiral pour ceux de bœufs Charolais $\left(\mathrm{E}_{25}, \mathrm{E}_{26}\right)$. Dans le cas des femelles, les résultats fournis par $\mathrm{X}_{1}$ et $\mathrm{X}_{2}$ semblent plus comparables, le tour spiral donnant en général de meilleures estimations du poids dans les races $\mathrm{Nor}$ mande et Pie noive, le tour de poitrine paraissant au contraire plus intéressant dans les races Pie rouge, Brune des Alpes, Salers et Limousine, c'est-à-dire dans les races de montagne et semi-montagne. Ces résultats ressortent clairement de l'examen du graphique 3 .

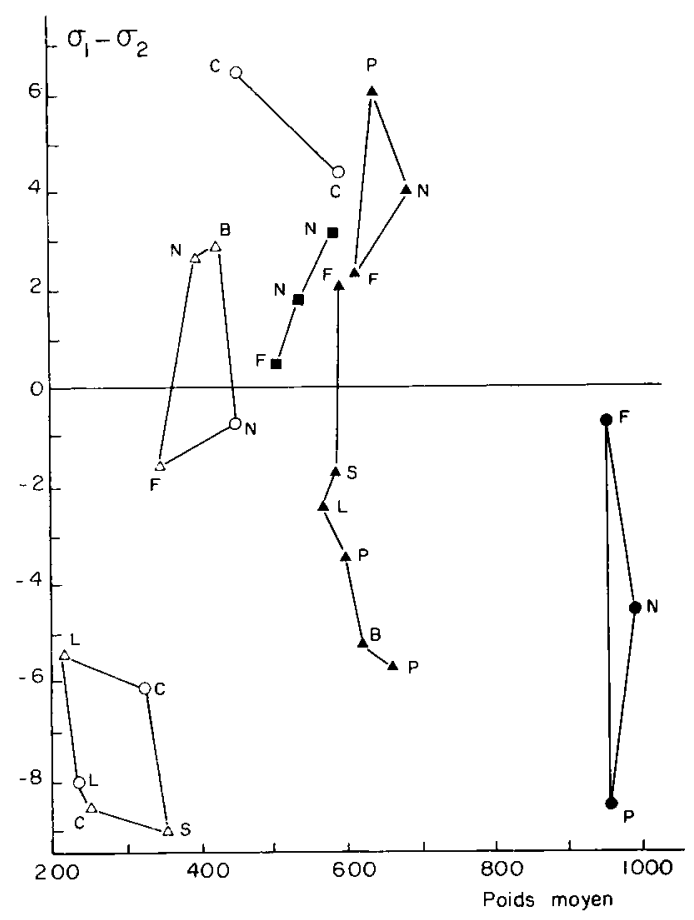

GRAPHULE 3. - Représentation schématique des différences suinant les principales caracléristiques des échantillons

(poids moyen, sexc, race, age)

$$
\begin{aligned}
& \mathrm{B}=\text { Brune des Alpes } \\
& \mathrm{F}=\text { Pie Noire } \\
& \mathrm{L}=\text { Limousine } \\
& \mathrm{N}=\text { Normande } \\
& \mathrm{P}=\text { Pie Ronge } \\
& \mathrm{C}=\text { Charolaise }
\end{aligned}
$$

Màles...........

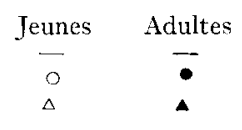

Comparaison des estimations $\widehat{Y}_{1}$ et $\widehat{Y}_{3}$.

L'incorporation du tour spiral dans une équation linéaire à 2 variables réduit l'écart-type de la distribution résiduelle de $\mathrm{I}$ à $22 \mathrm{~kg}$ suivant les cas. Cette réduction est surtout sensible pour les taureaux adultes ( 13 à $22 \mathrm{~kg}$ ), dans le cas des vaches adultes de concours ( 3 à $\mathrm{Io} \mathrm{kg}$ ) et pour les autres échantillons de femelles adultes ou en croissance. En ce qui concerne les veaux de moins d'un an, l'amélioration de précision apportée par l'utilisation du tour spiral est négligeable. 


\section{RECHERCHE D'UN TYPE D'AJUSTEMENT}

\section{Formules empiriques}

Nous avons d'abord étudié sur les échantillons $\mathrm{E}_{1}, \mathrm{E}_{\mathrm{q}}, \mathrm{F}_{33}$ l'efficacité statistique des 3 formules empiriques le plus couramment citées et employées :

$$
\begin{aligned}
& \text { formules de Crevat: } \begin{aligned}
Y & =80 \quad X_{1}^{3} \\
Y & =40 \quad X_{2}^{3} ;
\end{aligned} \\
& \text { formule de Quetelet: } Y=87,5 \quad X_{1}^{2} \times X_{5} .
\end{aligned}
$$

Cette efficacité est exprimée dans chaque cas par l'erreur moyenne, erreur dont la valeur tient compte à la fois des fluctuations systématiques et aléatoires de l'expression considérée autour du poids (tabl. 5).

$$
\text { TABIEAT } 5
$$

Erreurs moyennes liées à l'emploi des formules empiriques (kg)

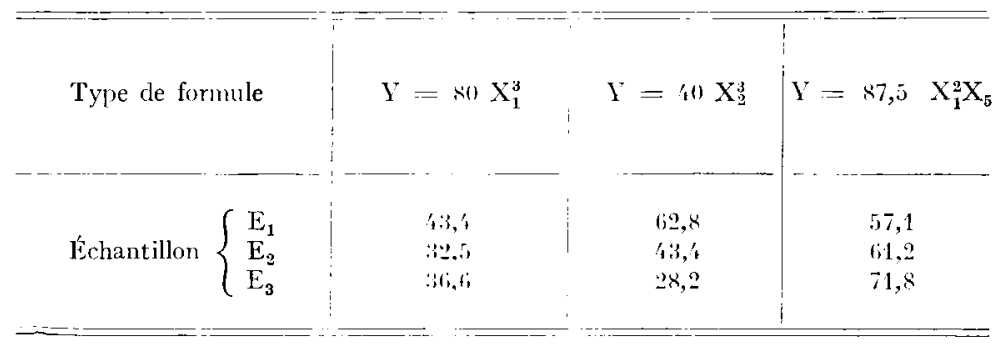

En dehors du fait évident que ces formules fournissent des erreurs nettement plus élevées que celles correspondant aux ajustements statistiques, il semble que la formule du Crevat $\mathrm{Y}=80 \mathrm{X}_{1}^{3}$ donne, dans l'ensemble, les meilleurs résultats.

$$
\begin{aligned}
& \text { Ajustements linéaires utilisant les puissances entières de } \\
& \qquad \mathrm{x}_{1} \text { et } \mathrm{x}_{2}
\end{aligned}
$$

La curvilinéarité des liaisons entre les mesures corporelles et le poids, dans les échantillons considérés en pratique, a poussé les chercheurs (JoHANsson et HII,DEMAN, I954) à rechercher des ajustements non linéaires et notamment de la forme :

$$
\mathrm{Y}=a \mathrm{X}_{i}^{n_{i}} \quad \text { ou } \quad a \prod \mathrm{X}_{i}^{n i}
$$

c'est-à-dire à ajuster des liaisons linéaires à partir des valeurs logarithmiques des variables.

Dans une précédente publication (VISSAC B., I959) nous avons montré à ce sujet, sur des données des échantillons I $4_{4}$ et I5 $_{5}$ qu'un ajustement linéaire de la forme :

$$
\mathrm{Y}=a \mathrm{X}_{1}{ }^{n}+b \mathrm{X}_{2}^{n^{\prime}}+c
$$


était aussi précis sinon plus que l'ajustement classique envisagé ci-dessus :

$$
\widehat{Y}=a \mathrm{X}_{1}^{n} \cdot \mathrm{X}_{2}{ }^{\prime} \text {. }
$$

Nous avons, dans cet esprit, étudié les ajustements à I ou 2 variables de la forme :

$$
\begin{aligned}
& \widehat{\mathrm{Y}}=a \mathrm{X}_{1}{ }^{n}+b \\
& \widehat{\mathrm{Y}}=a \mathrm{X}_{1}{ }^{n}+b \mathrm{X}_{2}{ }^{n^{\prime}}+c,
\end{aligned}
$$

avec $\mathrm{n}, \mathrm{n}^{\prime}$ entiers et tels que $3 \geqslant n, n^{\prime}>0$.

L'efficacité statistique de ces ajustements est exprimée par l'écart-type des différences $(\mathrm{Y}-\widehat{\mathrm{Y}})$, les valeurs obtenues sont présentées sur le tableau 6 ou les échantillons ont été rassemblés en 7 groupes :

$$
\begin{aligned}
& \mathrm{A}=\left(\begin{array}{lll}
\mathrm{E}_{12} & \text { à } & \mathrm{E}_{3}
\end{array}\right) \text { femelles adultes; } \\
& \mathrm{B}=\left(\mathrm{E}_{4} \text { à } \mathrm{E}_{6}\right) \text { taureaux ; } \\
& \mathrm{C}=\left(\begin{array}{lll}
\mathrm{E}_{7} & \text { à } & \mathrm{E}_{9}
\end{array}\right) \text { femelles adultes (poids de carcasse) ; } \\
& \mathrm{D}=\left(\mathrm{E}_{10} \text { à } \mathrm{E}_{13}\right) \text { veaux de moins d'un an; } \\
& \mathrm{E}=\left(\mathrm{E}_{14} \text { à } \mathrm{E}_{18}\right) \text { jeunes en croissance; } \\
& \mathrm{F}=\left(\mathrm{E}_{19} \text { à } \mathrm{E}_{124}\right) \text { fenelles adultes; } \\
& \mathrm{G}=\left(\mathrm{E}_{25} \text { et } \mathrm{E}_{26}\right) \text { bœufs. }
\end{aligned}
$$

Ajustements ne faisant intervenir qu'une seule variable.

La précision des ajustements n'est pas sensiblement améliorée par l'emploi de puissances de $\mathrm{X}_{1}$ ou $\mathrm{X}_{2}$ dans le cas des échantillons de femelles adultes $\left(\mathrm{E}_{1}\right.$ à $\mathrm{E}_{3}$ et $\mathrm{E}_{19}$ à $\mathrm{E}_{24}$ ) ; l'écart-type des différences n'est pas réduit dans 3 cas sur I.2 pour $\mathrm{X}_{2}$ et 7 cas sur $\mathrm{I} 2$ pour $\mathrm{X}_{1}$, dans les autres cas la réduction maximum enregistrée est de $0,64 \mathrm{~kg}$ pour $X_{2}\left(E_{21}\right)$ et $0,40 \mathrm{~kg}$ pour $X_{1}\left(F_{11}\right)$. I)ans le cas des échantillons de taureaux adultes, l'accroissement de précision est également faible $\left(0, \mathrm{I} 9 \mathrm{~kg}\right.$ pour $\mathrm{F}_{4}$ à $\mathrm{I}, 34 \mathrm{~kg}$ pour $\left.\mathrm{E}_{5}\right)$. Par contre, pour les jeunes en croissance, l'emploi des puissances d'ordre 2 ou 3 de $\mathrm{X}_{1}$ ou $\mathrm{X}_{2}$ améliore assez nettement la prédiction du poids. L'écarttype des erreurs observées dans le cas de la régression linéaire simple sur $\mathrm{X}_{1}$ et $\mathrm{X}_{2}$ est réduit dans 8 cas sur 9 pour $\mathrm{X}_{1}$ et dans 3 cas sur 9 pour $\mathrm{X}_{2}$ jusqu'à $4,08 \mathrm{~kg}$ pour $\mathrm{X}_{2}\left(\mathrm{E}_{15}\right)$ et $3,26 \mathrm{~kg}$ pour $\mathrm{X}_{1}\left(\mathrm{E}_{18}\right)$. I'une façon plus analytique pour les échantillons d'animaux en croissance, la meilleure estimation est obtenue pour $n=2$ dans le cas du tour de poitrine (6 cas sur 9) ; les résultats sont plus fluctuants dans le cas du tour spiral.

\section{Ajustements faisant intervenir 2 variables.}

Les résultats obtenus rejoignent cetxx relatifs aux ajustements ne faisant intervenir qu'une variable : si on se réfète comme base de comparaison à la précision des

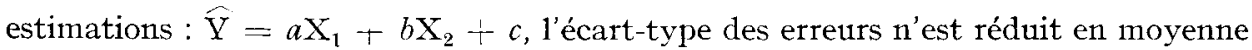
que de $0, I_{5} \mathrm{~kg}$ pour les femelles adultes et de $0,53 \mathrm{~kg}$ pour les taureaux adultes dans le cas du meilleur ajustement possible (o $<n \leqslant 3$, o < $n^{\prime} \leqslant 3$ ). Pour les échantillons de jeunes en croissance, la réduction moyenne de l'écart-type est plus appréciable : 2,6 $\mathrm{I} \mathrm{kg}$ (de $0,30 \mathrm{~kg}$ pour $\mathrm{E}_{1}$ à $5, \mathrm{I} 3 \mathrm{~kg}$ pour $\mathrm{E}_{5}$ ). 


\section{TABIEAU 6}

Écarts-types des distributions résiduelles

$$
\widehat{\mathrm{Y}}=a \mathrm{X}_{\mathrm{I}}^{n}+b \mathrm{X}_{2}^{n^{\prime}}+c
$$
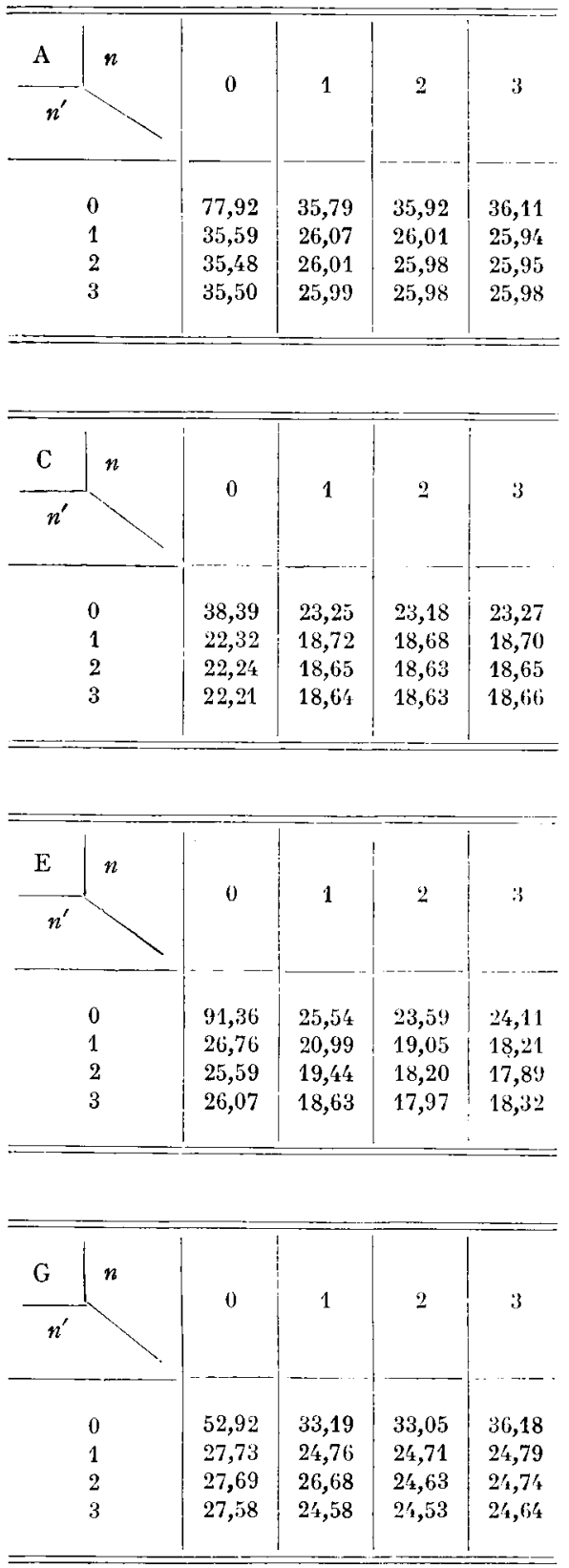
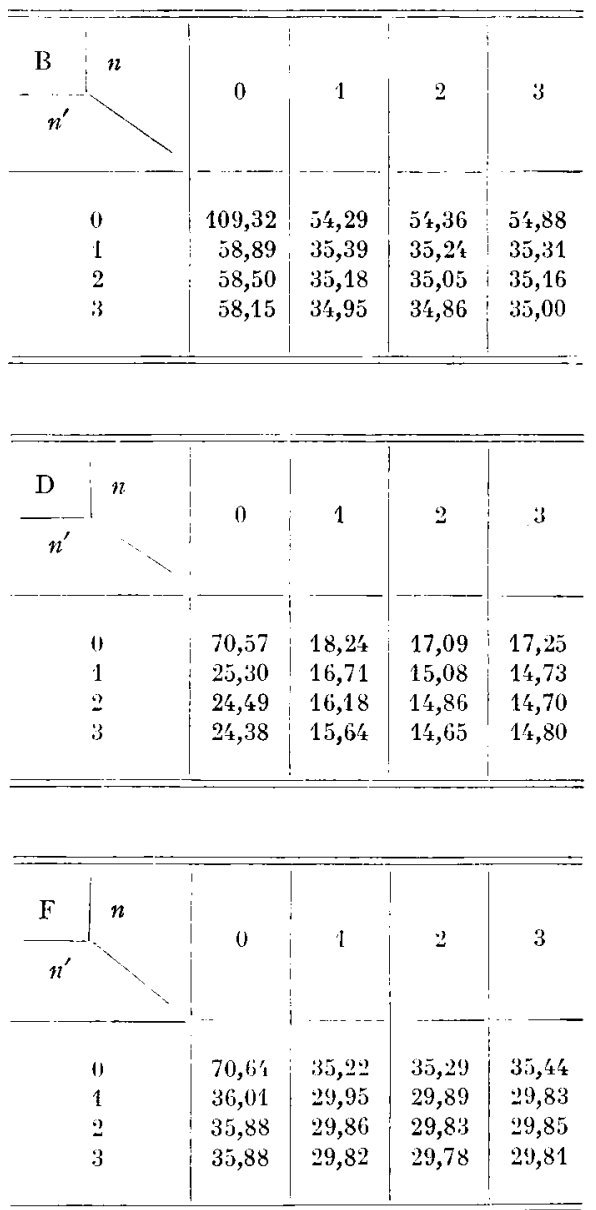
La meilleure estimation est obtenue pour ces échantillons de jeunes avec :

$$
\begin{aligned}
& n=2, \quad n^{\prime}=3 \text { pour } 4 \text { échantillons; } \\
& n=3, \quad n^{\prime}=3 \text { pour } 3 \text { échantillons; } \\
& n=\mathrm{I}, \quad n^{\prime}=3 \text { pour I échantillon; } \\
& n=3, \quad n^{\prime}=3 \text { pour I échantillon. }
\end{aligned}
$$

Si nous rassemblons les échantillons correspondants à des types d'animaux homogènes (vaches adultes, taureaux adultes, jeunes en croissance, bœufs à l'engrais) pour obtenir une estimée commune de l'erreur d'ajustement, la meilleure précision est toujours fournie par l'expression :

$$
\widehat{\mathrm{Y}}=a \mathrm{X}_{1}^{2}+b \mathrm{X}_{2}^{3}+c .
$$

Les variations de cette erreur d'ajustement avec $n$ et $n^{\prime}$ pour les échantillons de jeunes en croissance $\mathrm{E}_{10}$ à $\mathrm{E}_{18}$ sont représentées graphiquement (graph. 4).

Sur ce graphique la précision correspondant à une combinaison $n, n^{\prime}$ est exprimée verticalement par la différence entre l'erreur d'ajustement pour $n=\mathrm{I}$ et $n^{\prime}=\mathrm{I}$ et l'erreur d'ajustement pour les valeurs de $n$ et $n^{\prime}$ considérées.

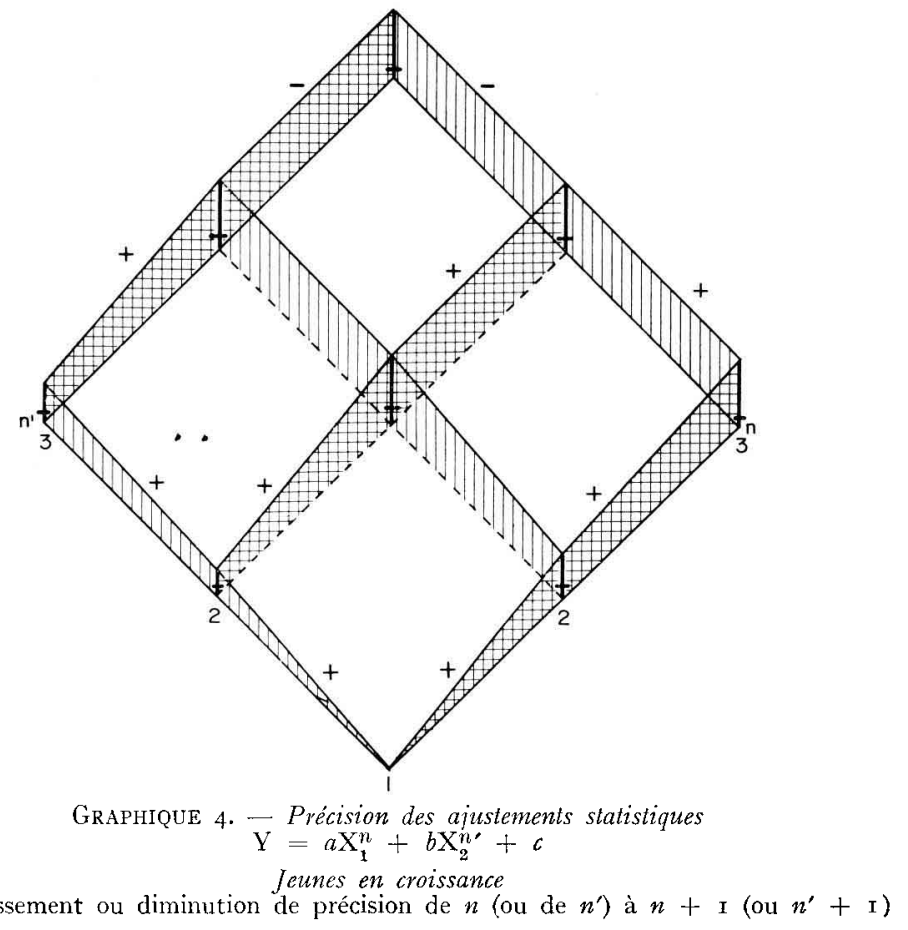

FACTEURS DE VARIATION DE PRÉCISION DE L'AJUSTEMENT IIÉS AUX CARAC'TÉRISTIQUES DE L'ÉCHANTILLON ANIMAL,

Il est évident que la précision des ajustements utilisés dépend largement de l'homogénéité des populations bovines auxquelles ils sont appliqués. Pour accroître cette précision on peut être conduit à établir une formule pour chaque type de bovin 
suivant sa race, son sexe, son état, son âge, etc. Toutefois, la multiplication des formules est incompatible avec l'utilisation pratique de la barymétrie qui doit rester simple. Le choix d'un "moyen terme " alliant la précision à la commodité nécessite de connaître :

$\mathbf{I}^{\circ}$ Le sens et l'importance des variations de l'ajustement statistique avec les caractéristiques de l'échantillon.

$2^{\circ}$ Les variations de précision de l'ajustement lorsque l'on tient compte de l'une ou de l'autre de ces caractéristiques en établissant plusieurs formules au lieu d'une seule.

Nous étudierons successivement les influences :

du sexe,

de la race,

cle l'âge et du format,

de l'état musculaire des animaux.

Ces études porteront sur certains échantillons ou sous-échantillons ci-dessus permettant de réaliser l'une ou l'autre de ces comparaisons; ces échantillons ou sous-échantillons ont été choisis de façon que les autres incidences étudiées ou les conditions expérimentales du contrôle puissent être considérées comme égales, en moyenne.

\section{Influence du sexe}

Nous avons comparé (tab1. 7) les valeurs des coefficients de régression partiels du poids vif sur le tour de poitrine $(a)$ et sur le tour spiral $(b)$ pour les échantillons de mâles et femelles :

Io d'animaux adultes des 3 races contrôlées au Concours général agricole de Paris $\left(\mathrm{E}_{1}\right.$ à $\left.\mathrm{E}_{6}\right)$;

TABLEAU 7

Comparaison des coefficients de régression partiels suivant le sexe

$\hat{\mathrm{Y}}=a \mathrm{X}_{1}+b \mathrm{X}_{2}+c$

\begin{tabular}{|c|c|c|c|c|c|c|c|}
\hline \multirow{2}{*}{ 岂 } & Coefficient & \multicolumn{2}{|c|}{ Míles } & \multicolumn{2}{|c|}{ Femelles } & \multicolumn{2}{|c|}{ Différence } \\
\hline & No echantil. & $a$ & $b$ & $a$ & $b$ & $a$ & $b$ \\
\hline 等 & $\begin{array}{l}E_{1}-E_{4} \ldots \\
E_{2}-F_{5} \ldots \\
E_{3}-E_{6} .\end{array}$ & $\begin{array}{l}5,318 \\
4,830 \\
6,976\end{array}$ & $\begin{array}{l}3,624 \\
4,577 \\
3,465\end{array}$ & $\begin{array}{l}3,860 \\
3,263 \\
6,804\end{array}$ & $\begin{array}{l}4,6 \times 6 \\
4,90 \% \\
3,394\end{array}$ & $\begin{array}{r}+1,158 \\
+1,567 \\
+\quad 2,172\end{array}$ & $\begin{array}{l}-1,062 \\
-0,327 \\
+0,111\end{array}$ \\
\hline 2 & $\begin{array}{l}E_{10}-E_{13} \\
E_{11}-E_{1} \\
E_{12}-E_{15}\end{array}$ & $\begin{array}{l}4,11: 3 \\
4,043 \\
3,320\end{array}$ & $\begin{array}{l}1,212 \\
0,376 \\
2,558\end{array}$ & $\begin{array}{l}8,625 \\
: 3,365 \\
2,4511\end{array}$ & $\begin{array}{l}0,206 \\
11,685 \\
: 3,106\end{array}$ & $\begin{array}{r}+0,512 \\
+\quad 0,678 \\
+\quad 0,870 \\
\end{array}$ & $\begin{array}{r}+1,006 i \\
-0,309 \\
-0,518 \\
\end{array}$ \\
\hline 桠 & $\begin{array}{l}E_{10}-E_{11}(100-200) \ldots \ldots \\
E_{10}-E_{11}(200-300) \ldots \ldots \\
E_{10}-E_{11}(300-400) \ldots \ldots\end{array}$ & $\begin{array}{l}2,56 \\
2,74 \\
1,86\end{array}$ & $\begin{array}{l}0,37 \\
0,44 \\
0,50\end{array}$ & $\begin{array}{l}2,05 \\
2,52 \\
2,80\end{array}$ & $\begin{array}{l}0,71 \\
0,6 ; 2 \\
11,11\end{array}$ & $\begin{array}{l}+0,51 \\
+0,22 \\
-0,91\end{array}$ & $\begin{array}{l}-0,36 \\
-0,18 \\
+\quad 0,39\end{array}$ \\
\hline
\end{tabular}


$2^{\circ}$ de jeunes en croissance des races Charolaise, Limousine et Normande;

$3^{\circ}$ dans le cas de la race Limousine, en séparant les veaux en 3 sous-échantillons :

$$
\begin{aligned}
& \text { veaux de I00 à I99 } \mathrm{kg} \text {; } \\
& \text { veaux de } 200 \text { à } 299 \mathrm{~kg} \text {; } \\
& \text { veaux de } 300 \text { à } 399 \mathrm{~kg} \text {. }
\end{aligned}
$$

Ces échantillons d'animaux mâles et femelles étaient, en outre, sensiblement comparables 2 à 2 du point de vue de l'âge moyen des animaux et de leur état d'engraissement, sauf en ce qui concerne les animaux de concours : 1'âge moyen des femelles étant, dans ce cas, supérieur à celui des mâles.

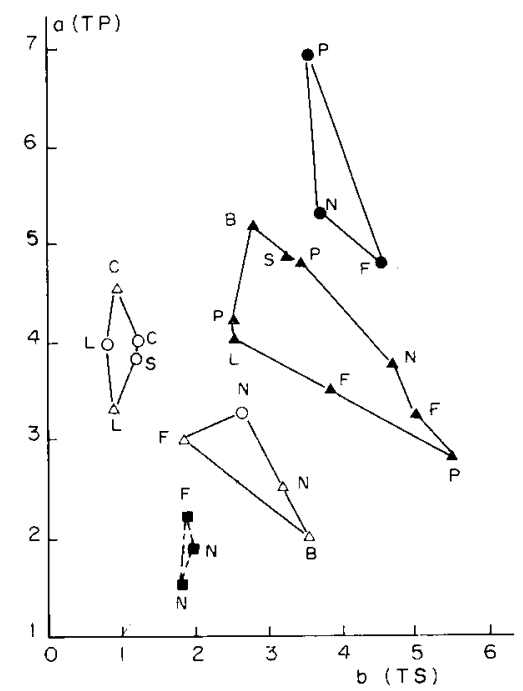

GRaringe 5. - Variations de a et b suivant les échantillons

$$
\widehat{\mathrm{Y}}=a \mathrm{X}_{1}+b \mathrm{X}_{2}+c
$$

- Poids de carcasse

$\mathrm{P}$ : Pie Rouge

$\mathrm{B}:$ Brune des Alpes

$\mathrm{F}$ : Frisonne Pie Noire

$\mathrm{X}$ : Vormante

5 : Salers

$\mathrm{C}$ : Chrolaise

I. : Limousine

D'une façon générale, il ressort que les valeurs de $a$ sont souvent supérieures pour les échantillons de mâles et inférieures pour les échantillons de femelles à celles trouvées pour $b$.

\section{Influence de la race}

En ce qui concerne l'influence de la race nous avons représenté graphiquement les variations conjointes de $a$ et $b$ suivant les échantillons (graph. 5). Il ressort 
de l'examen grossier de ce graphique qu'à part deux exceptions $\left(\mathrm{E}_{10}\right.$ et $\mathrm{E}_{21}$ : troupeaux expérimentaux) et à l'intérieur des 3 groupes d'échantillons :

$$
\begin{aligned}
& \text { jeunes en croissance (tous sexes); } \\
& \text { adultes (femelles); } \\
& \text { adultes (mâles). }
\end{aligned}
$$

les races se situent en 2 catégories :

- races laitières de plaine : Normande et Pie Noire à rapport $a / b$ faible;

- races laitières de montagne ou races à viande et rustiques : Pie Rouge, Brune, Salers, Limousine et Charolaise à rapport $a / b$ élevé.

La croissance du poids est donc dans ce dernier cas plus grande par rapport au tour de poitrine que par rapport au tour spiral, l'autre mesure étant considérée comme constante. C'est le contraire pour le groupe de nos 2 grandes races laitières de plaine. Ce fait est à rapprocher des différences de précision enregistrées ci-dessus, entre les races, suivant que l'on utilise le tour de poitrine ou le tour spiral : ce dernier est plus efficace dans le cas des races Normande et Pie Noire.

Il est intéressant de savoir dans quelle mesure l'emploi d'une formule par race améliore l'estimation du poids obtenue par une équation générale ne tenant pas compte de la race. Cette comparaison a été effectuée dans le cas particulier des échan-

\section{TABLEAU 8}

Variation de la précision statistique des ajustements linéaires

\begin{tabular}{|c|c|c|c|c|c|c|c|c|}
\hline \multicolumn{3}{|c|}{ Vaches $\left(E_{1}, E_{2}, E_{3}\right)$} & \multicolumn{6}{|c|}{ Taureaux $\left(\mathrm{E}_{1}, \mathrm{E}_{\overline{5}}, \mathrm{E}_{\overline{6}}\right)$} \\
\hline $\mathrm{X}_{1}$ & $\mathrm{X}_{2}$ & $X_{1}, X_{2}$ & $\mathrm{X}_{\mathrm{t}}^{*}$ & $\mathrm{X}_{1}^{* *}$ & $\mathrm{X}_{2}^{*}$ & $\mathrm{X}_{2}^{* *}$ & $\mathrm{X}_{1}, \mathrm{X}_{2}^{*}$ & $X_{1}, X_{2}^{* *}$ \\
\hline 2,84 & 0,71 & 2,13 & 7,50 & 7,98 & 9,76 & 1,90 & 2,52 & 2,73 \\
\hline
\end{tabular}

(Différence des écarts-types résiduels en kg)

A - Une formule par race

$\mathrm{B}$ - Une formule par classe de poids (taureaux $\mathrm{E}_{4}, \mathrm{E}_{5}, \mathrm{E}_{6}$ )

\begin{tabular}{c|c|c}
\hline & Une formule par race & Une formule générale \\
\hline $\mathrm{X}_{1}$ & 3,70 & \\
\hline $\mathrm{X}_{2}$ & 6,31 & 5,22 \\
\hline $\mathrm{X}_{1}, \mathrm{X}_{2}$ & 5,08 \\
\hline
\end{tabular}

* Une formule générale quel que soit le poids ** Une formule par classe de poirls 
tillons ci-dessus $\left(\mathrm{E}_{1}\right.$ à $\left.\mathrm{F}_{6}\right)$. L'écart-type des ajustements $(\mathrm{Y}-\overline{\mathrm{V}})$ est calculé globalement pour les 3 races en cause : successivement pour les vaches et les taureaux et en séparant, dans ce dernier cas, les animaux en classes de poids. L'amélioration obtenue dans ce cas particulier, est de 1'ordre de 2 à $3 \mathrm{~kg}$; (tabl. $8 \mathrm{~A}$ ).

\section{Influence de l'age et du format}

Le fait que les liaisons étudiées entre $Y, X_{1}$ et $X_{2}$ ne soient pas absolument linéaires, surtout pour les animaux en cours de croissance, peut s'expliquer si les coefficients de régression étudiés varient, pour un même type d'animal, avec le format et surtout avec l'âge.

Ces variations ont été précisées sur quelques exemples :

a) d'une façon analytique en constituant à l'intérieur de certains échantillons des sous-groupes où les animaux sont répartis par classes de poids:

$$
\begin{aligned}
& \text { échantillons } \mathrm{K}_{4} \text { à } \mathrm{E}_{6} \text { (taureaux adultes); } \\
& \text { échantillons } \mathrm{K}_{i_{17}} \text { et } \mathrm{E}_{13} \text {. }
\end{aligned}
$$

b) globalement, en comparant des échantillons de vaches et de génisses de la même race contrôlées simultanément dans les mêmes fermes et par les mêmes opérateurs :

$\begin{array}{ll}\text { Race Pie Noire } & \mathrm{E}_{17} \text { et } \mathrm{E}_{22} ; \\ \text { Race Limousine } & \mathrm{E}_{13} \text { et } \mathrm{E}_{23} \text {; } \\ \text { Race Salers } & \mathrm{E}_{18} \text { et } \mathrm{E}_{24} ; \\ \text { Race Brune des Alpes } & \mathrm{E}_{16} \text { et } \mathrm{E}_{19} .\end{array}$

I es coefficients de régression partiels $a$ et $b$ sont rassemblés sur le tableau 9 en vue des comparaisons analytiques et globales précisées ci-dessus.

Bien que les résultats ne soient pas systématiques, on enregistre en général, un accroissement des valeurs de $a$ et $b$ avec l'âge et le poids moyen des échantillons cette conclusion est surtout valable pour le tour de poitrine.

Dans le cas des échantillons $\mathrm{F}_{4}$ à $\mathrm{E}_{6}$, l'établissement des 2 équations de régression linéaires par classe de poids, au lieu d'une seule, réduit l'écart-type des erreurs intraraces de :

$$
\begin{aligned}
& +3,70 \mathrm{~kg} \text { pour } \mathrm{X}_{1} \\
& \left.+6,3 \mathrm{I} \mathrm{kg} \text { pour } \mathrm{X}_{2} \text { (tabl. } 8 \mathrm{~B}\right)
\end{aligned}
$$

Quant à 1'équation de régression à 2 variables, sa précision est améliorée, dans les mêmes conditions, de $+4,96 \mathrm{~kg}$. Ces chiffres correspondent à une réduction de la variance des erreurs sensiblement supérieure à celle que l'on obtiendrait sur les mêmes échantillons en établissant une formule par race au lieu d'une formule générale.

Pour les échantillons I2 et I3, l'établissement de 3 équations de régression linéaire à 2 variables $\left(\mathrm{X}_{1}, \mathrm{X}_{2}\right)$ par classe de poids au lieu d'une seule, permet d'abaisser l'erreur résiduelle de $I_{7,28} \mathrm{~kg}$ à $10,50 \mathrm{~kg}$ pour $\mathrm{E}_{12}$ et de $\mathrm{I}_{4,50}$ à $9,95 \mathrm{~kg}$ pour $\mathrm{E}_{13}$. 
TABLEAU 9

Comparaison des coefficients de régression a et $\mathrm{b}$ par classe de poids

$$
\widehat{\mathrm{Y}}=a \mathrm{X}_{1}+b \mathrm{X}_{2}+c
$$

Comparaisons analytiques

Échantillons $\mathrm{E}_{4}$ à $\mathrm{E}_{6}$ (taureaux)

\begin{tabular}{|c|c|c|c|c|c|c|c|c|}
\hline \multirow{2}{*}{$\mathrm{N}^{0}$} & \multirow{2}{*}{ échantillon } & \multirow{2}{*}{ Race } & \multicolumn{2}{|c|}{$a$} & \multicolumn{2}{|c|}{$b$} & \multicolumn{2}{|c|}{$\begin{array}{c}\text { Différences } \\
\times(-800+800)\end{array}$} \\
\hline & & & $-800 \mathrm{~kg}$ & $+800 \mathrm{kgr}$ & $-800 \mathrm{~kg}$ & $+800 \mathrm{~kg}$ & $a$ & $b$ \\
\hline & $E_{4}$ & Normande & 2,36 & 5,16 & 5,36 & 3,73 & $-2,80$ & $+1,63$ \\
\hline & $\mathrm{E}_{3}$ & Pie Noire & 4,71 & 4,79 & 2,33 & 4.39 & $-0,08$ & $-2,06$ \\
\hline & $\mathrm{E}_{6}$ & Pie Rouge & 3,52 & 7,50 & $3,2 !$ & 2,57 & $-2,98$ & $+0,72$ \\
\hline
\end{tabular}

Échantillons $\mathbf{E}_{12}$ et $\mathrm{E}_{\mathbf{1 3}}$ (veaux de moins d'un an)

\begin{tabular}{|c|c|c|c|c|c|c|c|c|c|c|c|}
\hline \multirow{3}{*}{$\begin{array}{c}\text { No } \\
\text { échant. }\end{array}$} & \multirow{3}{*}{ Race } & \multicolumn{2}{|c|}{ I } & \multicolumn{2}{|c|}{ II } & \multicolumn{2}{|c|}{ III } & \multicolumn{2}{|c|}{ Diff. I - II } & \multicolumn{2}{|c|}{ Diff. II - III } \\
\hline & & $100-$ & $0 \mathrm{~kg}$ & 200 & $0 \mathrm{~kg}$ & 300 & $\mathrm{~kg}$ & & & & \\
\hline & & $a$ & $b$ & $a$ & $b$ & $a$ & $b$ & $a$ & $b$ & $a$ & $b$ \\
\hline $\mathrm{E}_{12}$ & Limous. $\left(0^{*}\right)$ & 2,56 & 0,37 & 2,74 & $0,4^{\prime} t$ & 1,86 & $0, \overline{0} 0$ & $-0,18$ & $-0,07$ & $+0,88$ & $-0,00$ \\
\hline $\mathrm{E}_{13}$ & Limous. (9) & 2,05 & 0,71 & 2,52 & 0,62 & 2,80 & 0,11 & $-0,47$ & $+0,09$ & $-0,28$ & $+0 ; 51$ \\
\hline
\end{tabular}

Comparaisons globales

\begin{tabular}{|c|c|c|c|c|c|c|c|}
\hline \multirow[b]{2}{*}{ No échant. } & \multirow[b]{2}{*}{ Race } & \multicolumn{2}{|c|}{$a$} & \multicolumn{2}{|c|}{$b$} & \multicolumn{2}{|c|}{ Différence } \\
\hline & & Jeunes & Adultes & Jeunes & Adultes & $a$ & $b$ \\
\hline$E_{17}-E_{22}$ & Pie Noire & 3,01 & 3,51 & 1,74 & 3,84 & $-0,50$ & $-2,10$ \\
\hline $\mathrm{E}_{13}-\mathrm{E}_{23}$ & Limousine & 3,37 & 4,10 & 0,69 & 2,53 & $-0,73$ & $-1,84$ \\
\hline $\mathrm{E}_{18}-\mathrm{E}_{24}$ & Salers & 5,76 & 4,93 & 0,58 & 3,19 & $+0,83$ & $-2,61$ \\
\hline $\mathrm{E}_{16}-\mathrm{E}_{19}$ & Brune & 2,02 & 5,33 & 3,47 & 2,66 & $-3,31$ & $+0,81$ \\
\hline
\end{tabular}

Cette précision est supérieure à celle que l'on obtenait avec la meilleure équation de régression de la forme:

$$
\widehat{\mathrm{Y}}=a \mathrm{X}_{1}^{n}+b \mathrm{X}_{2}^{n \prime}+c
$$

établie pour l'ensemble de chaque échantillon ( $\mathrm{I} 3, \mathrm{I} 9 \mathrm{~kg}$ pour $\mathrm{E}_{12}$ et Ir,39 $\mathrm{kg}$ pour $\mathrm{E}_{13}$ ).

\section{Influence de l'état de l'animal}

L'étude de ce facteur a porté sur les échantillons $\mathrm{E}_{7}$ et $\mathrm{E}_{8}$ constitués de vaches Normandes et Pie Noires de réforme dont la qualité commerciale, très variable, était contrôlée par un expert à l'abattoir ; les animaux étaient répartis en 4 classes 
de qualité suivant le barême en usage pour ce type de bovin sur le marché de Paris.

Nous avons rassemblé sur le tableau ro les valeurs des coefficients de régression de $\mathrm{Y}$ sur $\mathrm{X}_{1}(a)$ et de $\mathrm{Y}$ sur $\mathrm{X}_{2}(b)$ pour chacun de ces échantillons. On observe que ces coefficients varient dans le même sens que la qualité commerciale des animaux, surtout dans le cas du tour de poitrine.

TABLEAU IO

Variation des coeffcients de régression avec la qualité commerciale

\begin{tabular}{|c|c|c|c|c|}
\hline \multirow{3}{*}{ No échantillon } & \multicolumn{2}{|c|}{$E_{7}$} & \multicolumn{2}{|c|}{$\mathrm{E}_{8}$} \\
\hline & \multicolumn{2}{|c|}{ Normande } & \multicolumn{2}{|c|}{ Pie Noire } \\
\hline & $a$ & $b$ & $a$ & $b$ \\
\hline ( 1 bonne & 3,40 & 2,53 & 3,87 & 2,67 \\
\hline Oualité 2 & 3,25 & 1,93 & 2,52 & 2,02 \\
\hline Qualite\{: 3 & 2,91 & 2,86 & 2,78 & 2,56 \\
\hline \& mauvais & 1,68 & 2,10 & 0,30 & 1,14 \\
\hline Total & 3,29 & 2,90 & 3,79 & 2,89 \\
\hline
\end{tabular}

PRÉCISION DE L'AJUSTEMENT. IMPORTANCE DES ERREURS DE MESURE

Nous envisagerons, à la lumière des résultats ci-dessus :

I ${ }^{0}$ la précision brute de $1^{\prime}$ ajustement linéaire à 2 variables ;

$2^{\circ} 1^{\prime}$ incidence des erreurs de mesure sur cette précision.

\section{Précision de l'ajustement}

Après avoir étudié analytiquement les méthodes d'estimation du poids à partir des mesures, il est intéressant de comparer globalement la précision des meilleures estimations retenues pour chacun des échantillons étudiés. Cette comparaison est difficile en raison des écarts d'âge, de format moyen, et de variabilité de ces échantillons. Pour en tenir compte nous avons représenté graphiquement 1'écart-type des erreurs en fonction du poids moyen $(\overline{\mathrm{Y}})$ (graph. 6 ) et de l'écart-type de ce poids $\left(\sigma_{y}\right)$ (graph. 7) sans toutefois tenir compte de l'âge.

Il ressort de ces graphiques que l'erreur exprimée par l'écart-type des différences $(\mathrm{X}-\widehat{\mathrm{Y}})$ est sensiblement une fonction linéaire du poids moyen des échantillons.

Cet écart-type est d'environ :

$35 \mathrm{~kg}$ pour les échantillons de taureaux (I ooo kg);

$25 \mathrm{~kg}$ pour les échantillons de vaches (600 kg);

I $8 \mathrm{~kg}$ pour les échantillons jeunes de I à 2 ans (400 $\mathrm{kg}$ ) ;

I4 $\mathrm{kg}$ pour les échantillons de veaux de moins d'un an (200 kg). 


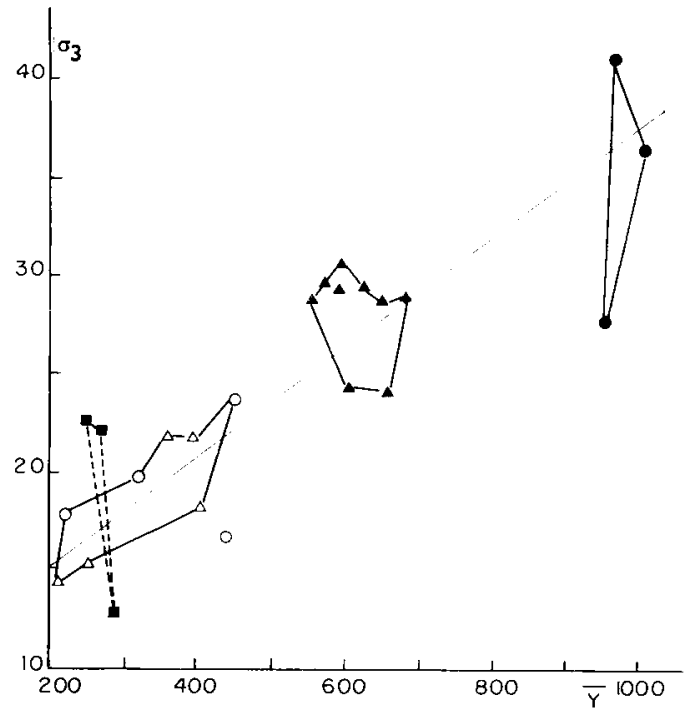

Graphique 6. - Liaison entre la précision de l'ajusiement $\widehat{Y}=\mathrm{a} X_{1}+\mathrm{b} X_{2}+\mathrm{c}$ et le poids moyen des échanillons $(\bar{Y})$

$\begin{array}{lll}\text { Mâles............ } & - & \circ \\ \text { Femelles } \ldots \ldots \ldots & \Delta & \bullet\end{array}$

- Poids de carcasse

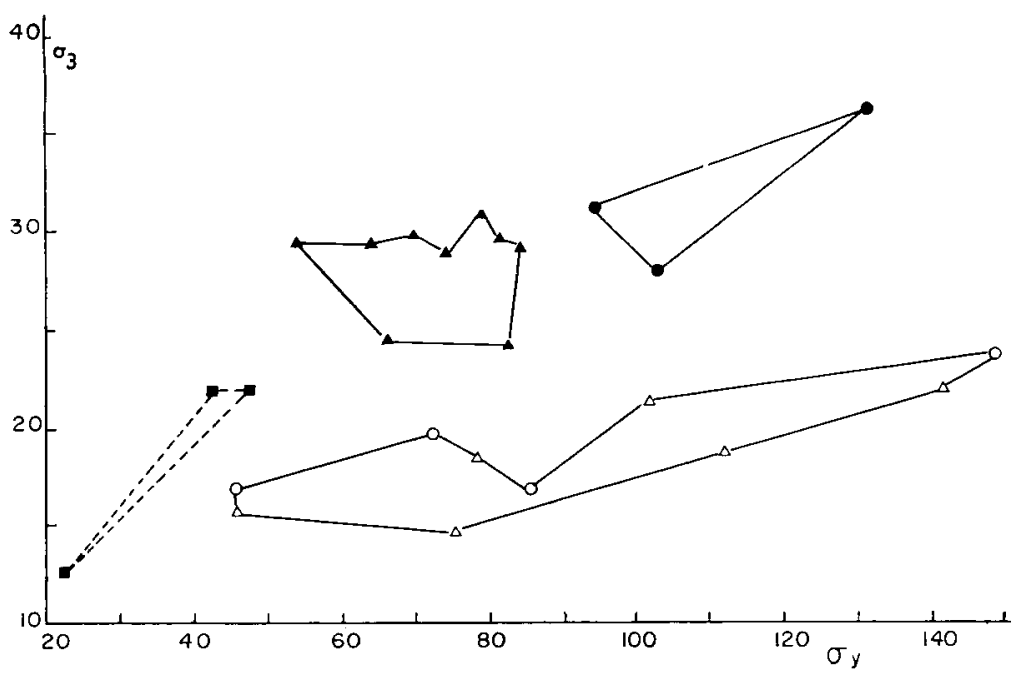

Graphique 7. - Liaison entre la précision de l'ajustement $\widehat{Y}=\mathrm{a} X_{1}+\mathrm{b} X_{\mathbf{2}}+\mathrm{c}$ et l'écart-type des poids des échantillons $\left(\sigma_{y}\right)$

$$
\text { Jeunes Adultes }
$$

Mâles..........

$\overline{0} \quad \bar{\bullet}$

Femelles........

- Poids de carcasse

Annales de Zootechnie. - I 966. 
L'écart-type des erreurs semble, par contre, dépendre peu de l'écart-type des échantillons étudiés si l'on divise ces derniers en 2 groupes :

- échantillons de bovins adultes;

- échantillons de bovins en croissance, à l'exception des échantillons d'animaux contrôlés à l'abattoir $\left(\mathrm{E}_{7}\right.$ à $\left.\mathrm{E}_{9}\right)$. La précision de l'ajustement apparaît meilleure dans le cas de bovins en croissance du fait de la grande variabilité de poids de ces échantillons.

\section{Analyse des erreurs d'ajustement}

Les erreurs d'ajustement calculées ci-dessus dépendent en fait, de 3 sources d'erreurs :

$$
\begin{aligned}
& \text { ajustement proprement dit; } \\
& \text { imprécision de la pesée; } \\
& \text { imprécision de la prise des mensurations. }
\end{aligned}
$$

Nous pouvons exprimer leur incidence sur 1'écart-type des différences $\mathrm{Y}-\widehat{\mathrm{Y}}$ en posant :

$$
\begin{aligned}
& \mathrm{Y}=y+\varepsilon_{0} \\
& \mathrm{X}_{1}=x_{1}+\varepsilon_{1} ; \\
& \mathrm{X}_{2}=x_{2}+\varepsilon_{2} ;
\end{aligned}
$$

$\mathrm{Y}, \mathrm{X}_{1}, \mathrm{X}_{2}$ étant les poids et mensurations contrôlés ; $y, x_{1}, x_{2}$ étant les poids et mesures réels de l'animal; $\varepsilon_{0}, \varepsilon_{1}$, $\varepsilon_{2}$ étant les erreurs de mesure.

$$
\begin{aligned}
\mathrm{Y}-\widehat{\mathrm{Y}} & =\mathrm{Y}-a \mathrm{X}_{1}-b \mathrm{X}_{2}-c \\
& =y-a x_{1}-b x_{2}-c+\varepsilon_{0}-a \varepsilon_{1}-b \varepsilon_{2}
\end{aligned}
$$

représentent l'incidence des 3 erreurs ci-dessus sur la différence $\mathrm{Y}-\widehat{\mathrm{Y}}$.

La variance des différences $(\mathrm{Y}-\widehat{\mathrm{Y}})$ peut être exprimée théoriquement :

$$
\sigma^{2}(\mathrm{x}-\hat{\mathrm{x}})=\sigma_{\mathrm{D}}^{2}+\sigma_{\varepsilon_{0}}^{2}+a^{2} \sigma_{\varepsilon_{1}}^{2}+b^{2} \sigma_{\varepsilon_{2}}^{2}
$$

en supposant que les erreurs sur les mesures et sur les poids sont indépendantes entre elles et indépendantes de 1'erreur réelle d'ajustement :

$$
\sigma_{\mathrm{D}}^{2}, \sigma_{\varepsilon_{0}}^{2} \text { et } a^{2} \sigma_{\varepsilon_{1}}^{2}+b^{2} \sigma_{\varepsilon_{2}}^{2}
$$

représentent la variance du poids due respectivement aux erreurs d'ajustement proprement dites, aux erreurs de pesée et aux erreurs de mesure.

$$
\text { Estimation de } \sigma_{\varepsilon_{1}}^{2} \text { et } \sigma_{\varepsilon_{2}^{2}}^{2} \text {. }
$$

Les valeurs de $\sigma_{\varepsilon_{i}}^{2}$ ont été estimées dans une étude sommaire de répétabilité portant sur 24 vaches Normandes $\mathrm{V}_{i}(i=I$... 24) mesurées successivement par 2 opérateurs $\mathrm{O}_{j}(j=I, 2)$ à l'aide de 2 types de rubans métriques :

un ruban en toile $\left(\mathrm{R}_{1}\right)$;

un ruban en courroie caoutchoutée $\left(R_{2}\right)$. 
Jans le cas du tour de poitrine $\left(\mathrm{X}_{1}\right)$, on a effectué 2 répétitions par opérateur et par animal; pour le tour spiral $\left(\mathrm{X}_{2}\right)$, par contre, on a tenu compte des différences pouvant intervenir d'un côté à un autre $\left.\mathrm{G}_{l}\right)(l=\mathrm{I}, 2)$ suivant que la mesure est prise :

$$
\begin{aligned}
& l=\mathbf{I} \text {, du sternum à l'épaule droite et à la hanche gauche ; } \\
& l=2 \text {, du sternum à l'épaule gauche et à la hanche droite. }
\end{aligned}
$$

Ces différences peuvent être liées notamment à la dissymétrie de position des organes internes des bovins.

Dans ce but les opérateurs travaillaient simultanément, l'animal étant mesuré 2 fois pour chacune des 4 positions définies sur le graphique 8 .
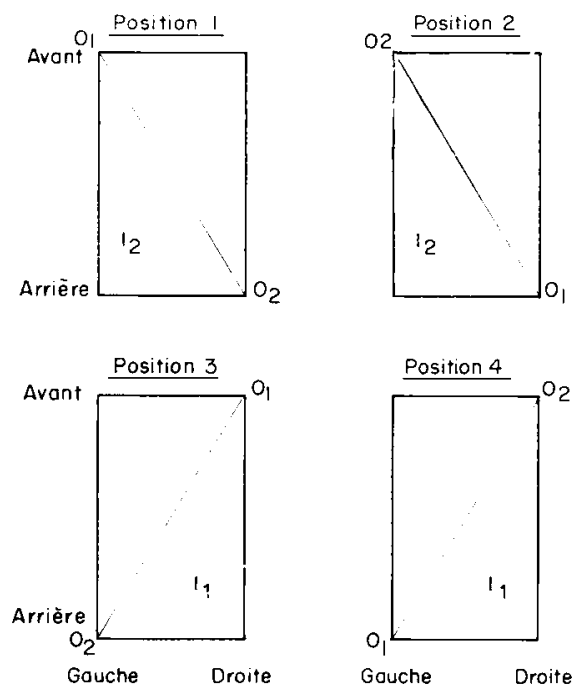

Graphique 8. - Schéma des 4 positions prises par 2 opérateurs $(O)$ pour la mesure du tour spiral $\left(X_{2}\right)$ sur un bonin

Les modèles théoriques correspondant à ces schémas peuvent s'exprimer :

$$
\begin{aligned}
& \mathrm{X}_{1}=\mu+v_{i}+o_{j}+(o v)_{i j}+\varepsilon_{i j n}, \\
& \mathrm{X}_{2}=\mu+v_{\imath}+o_{j}+c_{l}+(o v)_{i j}+(v c)_{i l}+(o c)_{j l}+\varepsilon_{i j l n} .
\end{aligned}
$$

Les diverses variables constituant ces modèles ( $\mu$ excepté) étant considérées comme des variables aléatoires, nous avons estimé pour chacune d'elles leurs variances :

$$
\sigma^{2}, \sigma_{o}^{2}, \sigma_{o v}^{2}, \sigma_{c}^{2}, \sigma^{2}{ }_{v c}, \sigma_{o c}^{2} .
$$

Le tableau II représente ces variances en p. Ioo de la variance totale :

$$
\begin{gathered}
\sigma^{2}=\sigma_{v}^{2}+\sigma_{o}^{2}+\sigma_{c}^{2}+\sigma^{2}{ }_{v o}+\sigma^{2}{ }_{v c}+\sigma_{o c}^{2}+\sigma_{\varepsilon}^{2} \\
\sigma_{v}^{2 \prime}=\frac{\sigma_{v}^{2}}{\sigma^{2}} ; \sigma_{o}^{2}=\frac{\sigma_{o}^{2}}{\sigma^{2}} \text { etc. }
\end{gathered}
$$

L'analyse de ces résultats fournit un certain nombre de conclusions :

- la mesure du tour de poitrine est plus précise que celle du tour spiral, ce qui justifie la technique opératoire adoptée : répétition de cette dernière mesure. 
L'incidence de ces erreurs de mesure sur l'erreur globale $\left(\sigma_{\mathrm{Y}}-\hat{\mathrm{Y}}\right)$ peut être estimée sur les échantillons de femelles adultes analogues à celui sur lequel a porté cette étude de répétabilité.

Avec $\quad a=4,5 ; \quad b=3,5 ; \quad \sigma_{\varepsilon_{1}}=\mathrm{I}, 2 ; \quad \sigma_{\varepsilon_{\mathrm{2}}}=\mathrm{I}, 6 ;$
$\sqrt{a^{2} \sigma^{2}{ }_{1}+b^{2} \sigma_{\varepsilon_{2}}^{2}}=7,78 \mathrm{~kg}$.

TABLEAU II

Composants de la variance

(en $\%$ de la variance totale)

\begin{tabular}{|c|c|c|c|c|c|}
\hline \multirow{2}{*}{\multicolumn{2}{|c|}{$\frac{\mathrm{X}_{i}}{\text { Ruban }}$}} & \multicolumn{2}{|c|}{$\mathrm{X}_{1}$} & \multicolumn{2}{|c|}{$\mathrm{X}_{2}$} \\
\hline & & \multirow{2}{*}{$\frac{\mathrm{R}_{1}}{96,6}$} & \multirow{2}{*}{$\begin{array}{r}\mathrm{R}_{2} \\
97,2\end{array}$} & \multirow{2}{*}{$\begin{array}{r}\mathrm{R}_{1} \\
88,9\end{array}$} & \multirow{2}{*}{$\begin{array}{r}\mathrm{R}_{2} \\
92,4\end{array}$} \\
\hline \multirow{8}{*}{$\sigma^{2^{\prime}}$} & $\sigma^{2^{\prime}} v$ & & & & \\
\hline & $\sigma^{2 \prime}{ }_{0}$ & 0,3 & 0,6 & 3,6 & 2,3 \\
\hline & $\sigma^{2^{\prime}}{ }_{c}$ & & & 0,1 & 0,8 \\
\hline & $\sigma^{2^{\prime}{ }_{v o}}$ & 0,0 & 0,0 & 1,2 & 0,5 \\
\hline & $\sigma^{2 \prime}{ }_{v e}$ & & & 3,3 & 1,2 \\
\hline & $\sigma^{2^{\prime}}{ }^{\prime}{ }^{\prime}$ & & & 0,5 & 0,2 \\
\hline & $\sigma_{\varepsilon}{ }^{2 \prime}$ & 3,11 & 1,8 & 2,5 & 2,6 \\
\hline & $\sigma_{\varepsilon}(\mathrm{cm})$ & 1,60 & 1,20 & 1,55 & 1,61 \\
\hline
\end{tabular}

TABLEAU I2

Erreur d'estimation du poids vif par les mensurations

\begin{tabular}{|c|c|c|c|}
\hline $\begin{array}{l}\text { Source } \\
\text { d'erreur }\end{array}$ & $\begin{array}{l}\text { Variance } \\
\qquad\left(\sigma^{2}\right)\end{array}$ & $\begin{array}{l}\text { Ecart-type } \\
(\mathrm{kg})\end{array}$ & $\begin{array}{l}\text { Variance en } \% \\
\text { de la variance } \\
\text { totale }\end{array}$ \\
\hline Erreur totale...... & 841 & 29 & 100,0 \\
\hline $\begin{array}{l}\text { Erreur de pesée. ........ } \\
\text { Erreur de mensuration... } \\
\text { Erreur d'ajustement pro- } \\
\quad \text { prement dite ........ }\end{array}$ & $\begin{array}{r}144 \\
61 \\
636\end{array}$ & $\begin{array}{l}12 \\
7,8 \\
25,2\end{array}$ & $\begin{array}{r}17,1 \\
9,3 \\
73,6\end{array}$ \\
\hline $\begin{array}{l}\text { Erreur d'ajustement }+ \\
\quad \text { erreur de mensuration. }\end{array}$ & 697 & 26,5 & 82,9 \\
\hline
\end{tabular}


Si avec les divers auteurs qui ont étudié la précision de la pesée, on estime à 2 p. Ioo du poids vif, l'erreur de pesée, lorsque cette dernière est effectuée à des heures variables du jour (Lush et COPEI.AND, I930), on a pour une vache de $600 \mathrm{~kg}$ :

$$
\sigma_{\varepsilon_{0}}=\mathrm{I} 2 \mathrm{~kg}, \quad \sigma_{\varepsilon_{0}}^{2}=\mathrm{I} 44 \mathrm{~kg} .
$$

L'erreur globale moyenne pour des échantillons de femelles adultes $\left(\sigma_{\mathrm{Y}}-\hat{\mathrm{x}}\right)$ $=29 \mathrm{~kg}$ ) peut donc être répartie en valeur absolue et relative (tabl. I2).

I a variance des erreurs dépend donc, pour les $3 / 4$ des erreurs d'ajustement, pour I/4 seulement de l'incidence des erreurs de mesure ou de pesée.

\section{Intérêt comparatif des estimations directe et indirecte du poids}

En retenant les chiffres du tableat1 $\mathrm{I} 2$, il apparaît que l'écart-type des erreurs. d'estimation du poids est environ 2 fois plus élevé dans le cas d'une mesure indirecte: $(26,5 \mathrm{~kg}$ contre I2 $\mathrm{kg})$ que dans le cas d'une mesure directe à heures variables. Le rapport serait de I à 4 dans le cas de pesées effectuées à heures fixes.

Si on considère que les objectifs du contrôle d'aptitude sont, bien souvent, d'estimer des valeurs moyennes, par ferme, par régime alimentaire, par type génétique, plus que des valeurs individuelles, on est condluit à comparer la valeur relative des méthodes d'estimation directe et indirecte pour estimer les poids moyens de lots d'animaux. On peut notamment se demander s'il n'est pas plus précis et moins onéreux d'utiliser une estimation indirecte sur un nombre élevé d'animaux (erreur de mesure élevée mais erreur d'échantillonnage moins importante). Si nous caractérisons par $\sigma_{p}^{2}$ la variance du poids d'un lot d'animaux, $\sigma_{\varepsilon_{0}}^{2}$ et $\sigma_{\varepsilon}^{2}$ les erreurs d'estimation directe et indirecte (voir ci-dessus), $n_{1}$ et $n_{2}$ les effectifs d'animaux des lots soumis à la pesée et à la barymétrie, une précision équivalente d'estimation du poids moyen sera obtenue si $n_{1}$ et $n_{2}$ sont tels que :

c'est-à-dire si

$$
\begin{gathered}
\frac{\sigma_{p}^{2}+\sigma_{\varepsilon_{0}}^{2}}{n_{1}}=\frac{\sigma_{p}^{2}+\sigma_{\varepsilon}^{2}}{n_{:-2}} \\
n_{\mathrm{I}}=\frac{\sigma_{p}^{2}+\sigma_{\varepsilon_{0}}^{2}}{\sigma_{p}^{2}+\sigma_{\varepsilon}^{2}} .
\end{gathered}
$$

Une comparaison plus réaliste de l'efficacité des 2 méthodes de détermination. du poids pourrait être effectuée si on pouvait exprimer en unités économiques, d'une part la précision, d'autre part les difficultés d'utilisation de l'un et de l'autre système. Une telle étude est possible si on applique le contrôle du poids à la sélection des taureaux sur le poids de leurs produits à un âge de référence (contrôle de descendance).

La précision de la méthode modifie l'erreur d'estimation du phénotype moyen des descendants (P) d'un taureau, elle intervient donc sur la précision de connaissance du génotype de ce dernier $(\mathrm{G})$ et sur le progrès génétique attendu par animal et par génération $(\Delta G)$ :

$$
\Delta \mathrm{G}=i \mathrm{R}_{p g} \sigma_{g}
$$

¿ : sélection différentielle;

$\sigma_{g} \quad$ : écart-type génétique ;

$\mathrm{R}_{p g}$ : coefficient de corrélation génotype-estimation du génotype. 
Les difficultés de détermination du poids s'expriment par le coût du contrôle : on peut considérer ce dernier $(\mathrm{F})$ comme proportionnel, dans un essai de testage, à l'effectif d'animaux contrôlés par taureau $(n)$ et au nombre de taureaux (v).

$$
\begin{aligned}
& \mathrm{F}=\mathrm{K} n v ; \\
& \mathrm{K}=\text { cô̂t unitaire du contrôle. }
\end{aligned}
$$

Pour obtenir dans un testage portant sur un même lot de taureaux des valeurs équivalentes du progrès génétique après estimation directe $\left(\Delta \mathrm{G}_{1}\right)$ ou indirecte $\left(\Delta \mathrm{G}_{2}\right)$ du poids, les effectifs $\left(n_{1}\right.$ et $\left.n_{2}\right)$ des lots de testage correspondants sont tels que :

$$
\frac{n_{1}}{n_{2}}=\frac{\sigma_{p}^{2}+\sigma_{\varepsilon_{0}}^{2}-0,25 \sigma_{g}^{2}}{\sigma_{p}^{2}+\sigma_{\varepsilon}^{2}-0,25 \sigma_{g}^{2}}
$$

cette expression est identique à la précédente, la variance phénotypique étant remplacée par la variance intra-taureau :

$$
2\left(\sigma_{p}^{2}-0,25 \sigma_{g}^{2}\right) .
$$

D'après les valeurs de $\sigma_{\varepsilon_{0}}^{2}$ et $\sigma_{\varepsilon}^{2}$ obtenues ci-dessus et en posant $\sigma_{p}^{2}=4900$ (chiffre moyen correspondant aux échantillons de femelles adultes de cette étude) on obtient $\frac{n_{1}}{n_{2}}=0,90$ pour des valeurs du coefficient d'héritabilité $\left(h^{2}=\frac{\sigma_{g}^{2}}{\sigma_{p}^{2}}\right)$ très variables.

Les rapports des frais de testage correspondant aux 2 systèmes mis en comparaison sont :

Ces frais seront égaux si :

$$
\frac{\mathrm{F}_{1}}{\mathrm{~F}_{2}}=\frac{\mathrm{K}_{1} n_{1}}{\mathrm{~K}_{2} n_{2}}
$$

$$
\mathrm{K}_{2}=0,90 \mathrm{~K}_{1}
$$

c'est-à-dire si le coût unitaire d'application de la barymétrie est inférieur de Io p. Ioo au coût de la pesée. Les valeurs relatives de ces coûts sont en fait variables en pratique suivant de nombreux facteurs et notamment :

- l'effectif d'animaux contrôlés par ferme ;

- 1a répartition dans le temps et dans l'espace des contrôles;

- le mode d'élevage des animaux: en liberté ou en stabulation entravée.

\section{IV. - DISCUSSION}

Nous discuterons successivement les 4 points abordés dans cette étude à la lumière des données bibliographiques récentes et de la synthèse des travaux plus anciens présentés par JoHANSSON et HII,DEMAN (I954).

\section{Choix des mesures}

L'intérêt du tour de poitrine $\left(\mathrm{X}_{1}\right)$ et du tour spiral $\left(\mathrm{X}_{2}\right)$ pour prédire le poids ressort de notre étude. La plupart des auteurs qui se sont intéressés à la barymétrie ont effectivement choisi le tour de poitrine comme critère essentiel d'estimation du 
poids, le tour spiral étant néanmoins retenu par certains : MARCQ et LAHAYE (I935).

Le fait que le tour spiral fournisse un ajustement moins précis que le tour de poitrine pour les jeunes et plus précis au contraire pour les adultes peut recevoir 2 interprétations différentes :

- L'erreur de mesure du tour spiral est proportionnellement élevée pour les jeunes par rapport à celle du tour de poitrine; nous avons effectivement pu vérifier que le rapport de la variance relative (coefficient de corrélation intra-classe) des erreurs de mesure du tour spiral sur la variance des erreurs de mesure du tour de poitrine était de 2 à 3 fois plus élevée chez les jeunes veaux de moins d'un an que chez les vaches adultes (Rouvier, données non publiées). On conçoit, en effet, que si le tour de poitrine est aussi commode à réaliser sur les bovins quel que soit leur âge, la mesure précise du tour spiral exige une immobilité des animaux et une rigidité corporelle que ne possèdent pas les jeunes animaux ;

- Bien qu'il ne nous soit pas possible de fournir une justification objective de cet argument, il est possible que le tour spiral rende mieux compte que le tour de poitrine de l'importance de parties corporelles dont le développement est plus tardif: allongement du corps, masses musculaires postérieures notamment. C'est peut-être également en faisant appel à des différences de variabilité morphologique entre les 2 races laitières du nord de la France (Pie Noire et Normande) d'une part, et les races de semi-montagne du Centre et de l'Eist (Pie Rouge, Salers, Limousine, Charolaise) d'autre part, qu'on pourrait interpréter l'intérêt plus grand du tour spiral dans le premier groupe.

Quoi qu'il en soit, si on excepte les veaux de moins d'un an ( $\left(\mathrm{L}_{10}\right.$ à $\left.^{\mathrm{L}_{13}}\right)$, l'emploi des 2 mesures retenues (tour de poitrine et tour spiral) dans une équation à 2 variables apporte un accroissement notable de précision par rapport à chacun des deux ajustements simples (tour de poitrine ou tour spiral) ; cela est contraire à l'opinion de SteEnsberg (I925), Hansson (I926), HVidsten (I940), Johansson et Hilideman (I954) qui indiquent que le poids vif n'est pas estimé avec plus de précision par plusieurs mesures incluant le tour de poitrine que par le tour de poitrine seul. A l'inverse, beaucoup de chercheurs ont cru bon d'associer au tour de poitrine une mesure complémentaire qui est, généralement, la longueur totale : MARCQ et all. (I953), Ross (I958), SzczEKIN KRotow (I96I), BURT (I957) ou le tour ventral BURT (I957). Ces mesures rendent alors compte soit de 1'allongement du corps, soit de l'importance du ventre. Il reste que l'intérêt d'adjoindre au tour de poitrine une deuxième mesure est aussi fonction des difficultés de réalisation : cette remarque est importante dans le cas du tour spiral.

L'adjonction d'une troisième mesure au tour de poitrine et au tour spiral dans une équation à trois variables n'est efficace, quoique très légèrement, qu'avec la largeur de poitrine $\left(\mathrm{X}_{8}\right)$ : l'emploi d'ajustements à trois variables est d'ailleurs rarement retenu : BURT (1957).

\section{Recherche d'un type d'ajustement}

Les formules proposées par les divers auteurs tiennent toutes compte du fait que, la signification géométrique du poids (volume) et des mensurations (longueur) étant différente, leurs variations simultanées ne peuvent s'exprimer par une relation linéaire ; BRODY (I945). 
Les solutions adoptées par les auteurs peuvent être classées sous 3 rubriques :

$I^{0}$ ajustements linéaires à partir des valeurs logarithmiques;

$2^{\circ}$ ajustements linéaires à partir des puissances entières des mesures ;

$3^{\circ}$ ajustements linéaires par catégorie d’animaux, mais pour des lots d'âge homogène.

Ajustements linéaires à partir des valeurs logarithmiques des mesures.

A la suite de Davis et all. (I937), Hvrdsten (I940), Auriol et Duplan (I960), BAGOT (I956) ont utilisé cet ajustement à partir du tour de poitrine ; le coefficient trouvé pour $n$ dans l'équation :

$$
\mathrm{Y}=a \cdot \mathrm{X}_{1}^{n}
$$

était dans tous les cas égal ou légèrement inférieur à 3. Si cet ajustement présente l'intérêt d'exprimer la relation : poids vif-masse corporelle pour des populations bovines d'âges disparates, il n'élimine pas pourtant la curvilinéarité des liaisons et l'incidence sous-jacente de l'âge ; BroOKEs et HARRINGTON (I960). STEENSBERg et OSTERGAARD (I945), BURT (I957) proposent d'ailleurs de tenir compte de l'âge dans une équation de régression de la forme : $\mathrm{Y}=a \mathrm{X}_{1}{ }^{n} \mathrm{~A}^{m}$.

Ajustements linéaires à partir des puissances des mensurations.

Cette méthode est utilisée notamment par HvidsTEN (1940) qui propose une expression du type : $\widehat{\mathrm{Y}}=a \mathrm{X}_{1}+b \mathrm{X}_{1}^{3}$. Johansson et HildDEMan (I954) de leur côté déterminent sur jeunes bovins une équation de la forme :

$$
\widehat{\mathrm{Y}}=a \mathrm{X}_{1}^{2}+b \mathrm{X}_{1}+c .
$$

On peut rattacher à cette méthode, l'expression préconisée par BuRT (I957) à partir de la longueur du corps $\left(\mathrm{X}_{5}\right)$ du tour de poitrine $\left(\mathrm{X}_{1}\right)$ et du tour ventral $\left(\mathrm{X}_{11}\right)$;

$$
\widehat{\mathrm{Y}}=a \mathrm{X}_{5}\left(\mathrm{X}_{1}^{2}+\mathrm{X}_{11}^{2}+\mathrm{X}_{1} \mathrm{X}_{11}\right)+b \text {. }
$$

Dans ce type d'ajustement, les auteurs incluent un terme constant qui ne figure pas dans l'ajustement précédent où la courbe obtenue doit passer par le point de coordonnées ou soit considèrent en les additionnant, des termes comportant les puissances entières de une ou plusieurs variables.

L'expression que nous avons retenue personnellement à la suite d'études antérieures :

$$
\widehat{\mathrm{Y}}=a \mathrm{X}_{1}^{n}+b \mathrm{X}_{2}^{n \prime}+c\left(o \leqslant n, n^{\prime} \leqslant 3\right)
$$

permet d'obtenir pour $n$ et $n^{\prime}$ des valeurs voisines de celles fournies par l'ajustement linéaire sur les valeurs logarithmiques :

L'intérêt d'un tel ajustement, à partir de puissances des mesures supérieures à I et par rapport à l'ajustement linéaire simple n'est sensible que pour des échantillons d'animaux en période de croissance et d'âges très variés.

Ajustements linéaires simples par catégorie d'animaux.

I'imprécision des ajustements ci-dessus tenant pourtant compte de la curvilinéarité des liaisons a conduit certains auteurs à comparer la précision qu'ils four- 
nissent à celle que l'on obtiendrait par une succession de régressions linéaires établies par classe de valeurs des mesures ou du poids.

HVidsTen (I940) trouve à ce sujet qu'un ensemble de 2 droites de régression établies pour 2 classes d'animaux d'après les valeurs du tour de poitrine donne de meilleurs résultats que les ajustements curvilinéaires sur les puissances de $\mathrm{X}_{1}$; Johansson et Hildeman (I954), de leur côté, optent pour une série de droites de régression, par classe d'âge, de préférence à une régression curvilinéaire établie sur un échantillon d'âges variés.

C'est un résultat équivalent que nous obtenons dans notre travail sur les échantillons $\mathrm{I}_{4}$ à $\mathrm{E}_{6}$ notamment, pour lesquels l'établissement de 2 droites de régression par échantillon dans le premier cas, suivant la valeur du poids, fournit des ajustements meilleurs que l'équation du type :

$$
\widehat{\mathrm{Y}}=a \mathrm{X}_{1}^{\prime \prime}+b \mathrm{X}_{2}^{\prime \prime}+c,
$$

établie sur l'ensemble de chaque échantillon.

On est ainsi conduit à étudier d'une façon plus analytique le sens des variations du poids en fonction des mensurations et plus particulièrement du tour de poitrine suivant les divers facteurs nutritionnels, génétiques qui peuvent intervenir sur cette relation.

\section{Facteurs de variation de l'ajustement}

\section{Influence de l'âge.}

Nous avons vu ci-dessus que cette influence a été prise en considération par certains auteurs, soit lorsque l'âge est inclus dans la formule barymétrique, STEENsBERG et OSTERGAARD (I945), soit indirectement lorsque l'on établit des équations de régression par classe de valeurs du poids ou d'une mesure.

DAVIs et all. (I937) indiquent que cette influence est fondamentale: l'écart-type des erreurs est réduit de moitié quand on établit des équations curvilinéaires par classe d'âge au lieu d'une équation générale. Pour Johaxsson et Hildeman (I954), le coefficient de régression du poids sur le tour de poitrine augmente avec l'âge jusqu'au vêlage (intra-race et classe d'état musculaire). Par contre, pour des femelles adultes, ces auteurs n'observent pas de différence entre les valeurs de ce coefficient, intra-classes d'âge et entre classes, pour 2 races suédoises. Mougin et Auriou (I960) obtiennent de leur côté des coefficients de régression voisins pour des vaches Pie Rouge réparties en 2 classes d'âge.

BERGE (I953), par contre, établit une table de correspondance : poids, tour de poitrine en répartissant ses animaux en 3 classes d'après leur dentition. Pour BurT (I957), sur des vaches également, l'adjonction de l'âge au tour de poitrine entraîne une diminution significative mais faible de ( 2 à 2 I p. IOo) de la variance résiduelle. Cet avis semble partagé par St'teEnsberg et OstergaArd (I945).

\section{Infuence du sexe.}

Cette influence est en fait difficilement séparable d'autres incidences, nutritionnelles en particulier : les mâles et les femelles n'étant pas entretenus de façon similaire. JOHANSSON et HIr,DEMAN (I954) trouvent des coefficients de régression du poids 
vif sur le tour de poitrine plus élevés pour les mâles. Dans notre étude, l'incidence apparemment plus importante du tour de poitrine par rapport au tour spiral chez les mâles pour prédire le poids, peut s'interpréter par une différence relative d'allométrie du poids par rapport à ces 2 mesures entre les mâles et les femelles. Nous pensons surtout que ceci doit être lié au meilleur état musculaire des échantillons de bovins mâles; cette situation se caractérise, comme nous l'avons vu (tabl. II) par un coefficient de régression partiel du poids sur le tour de poitrine proportionnellement plus élevé et inversement.

\section{Influence de la race.}

Les variations d'origine génétique (race, génotype) de la relation poids-tour de poitrine ne paraissent pas notables entre des races de types différents, BroOKES et HARRINGTON (I960), JoHANSSON et HILDEMAN (I954), a fortiori entre descendance de taureaux, Johansson et HILDEMAN (I954).

\section{Influence de l'état musculaire.}

L'incidence importante du niveau nutritionnel et, par voie de conséquence, de l'état des animaux sur la relation poids-tour de poitrine a été soulignée par divers auteurs. BroOKEs et HARRINGTON (I960) estiment même que les variations de régime alimentaire rendent la barymétrie difficilement utilisable. L'incidence du régime alimentaire sur les relations poids-tour de poitrine a été signalée par JoHANsSON et HILDEMAN (I954), BERGE (I953), STEENSBERG et OSTERGAARD (I945) notamment.

La diminution des coefficients de régression de $\mathrm{Y} / \mathrm{X}_{\mathbf{1}}$ avec celle de l'état des animaux est confirmée dans notre travail ; il est toutefois intéressant de noter l'incidence plus grande des variations du tour de poitrine sur le poids pour des animaux en bon état ; cette mesure devrait donc être, semble-t-il, un critère de l'état d'engraissement des animaux meilleur que le tour spiral.

Parmi les autres influences étudiées par les auteurs, il convient de signaler l'état de gestation des animaux. Les valeurs de poids sont généralement sous-estimées en fin de gestation; Hvidstein (I940), OstergaArd (I956). Mougrn et AUriol, (I960) proposent d'utiliser en race Montbéliarde une équation de régression pour vaches gestantes et une pour les autres: le coefficient de régression du poids sur le tour de poitrine étant supérieur dans le premier cas. HvIDSTEN (I940) fait remarquer que le tour de poitrine augmente de $2 \mathrm{~cm}$ pendant la gestation, cette variation correspondant à une amélioration de l'état des animaux; il ne pense pas utile, par contre, d'apporter un facteur de correction dans la mesure où on admet que le poids obtenu par ajustement fournit non le poids réel des animaux mais ce poids diminué du contenu de l'utérus.

\section{Précision de l'estimation du poids.}

La précision des ajustements obtenus à partir du tour de poitrine et du tour spiral ( 4 à 7 p. roo du poids vif moyen suivant les échantillons) est identique ou inférieure d'une façon générale à celle obtenue par les divers auteurs à partir du seul tour de poitrine : 6,2 p. IOO par HANSSON (I926), 5,05 p. IOO par BRANTON et SALISBURY (I946), 4,97 p. IOO par SLAGSVOLD (I949), 6 p. IOo par JoHANSSON et HIIDEMAN (I954), 6,3 à 6,5 p. IOO par HVidSTEN (I940), I3 p. IOO par DAvis et all. (I937) sur un échantillon, il est vrai, très hétérogène. 
Si la précision des ajustements, exprimée en valeur absolue, augmente avec le poids moyen des échantillons, elle diminue quand on la considère en p. Ioo du poids moyen de $7 \mathrm{p}$. Ioo pour les veaux à $3,5 \mathrm{p}$. Ioo pour les taureaux; cela est en accord avec les résultats de Johansson et Hildeman (I954).

Les erreurs de mesure du tour de poitrine et du tour spiral qui figurent dans notre travail ( $\mathrm{I}, 2$ à $\mathrm{I}, 6 \mathrm{~cm}$ ) soit environ 0,6 à 0,8 p. Ioo des mesures, sont équivalentes à celles signalées jusqu'ici pour le tour de poitrine : $0,59 \mathrm{p}$. Ioo par JoHANsSON et VENGE (I95I), 0,8 à 0,9 p. IOO pat LuSH et Copki,AND (I930). Eńn ce qui concerne le tour spiral deux faits sont à souligner :

- l'existence de différences entre opérateurs suivant que ces derniers sont placés à l'avant ou à l'arrière des animaux ;

- la présence d'une interaction animal-côté, interaction qui peut s'expliquer par une dissymétrie caractéristique de chaque animal, fonction de son état de gestation ou de l'état de réplétion de sa panse.

Ces faits conduisent à conseiller la réalisation de deux mesures par animal : une par côté, avec interversion de l'une à l'autre des opérateurs situés à l'avant et à 1 'arrière de l'animal.

L'analyse des erreurs d'ajustement nous conduit à des résultats sensiblement voisins de ceux rapportés par HVIISTEN (I940) : 7,8 $\mathrm{kg}$ contre 8,3 $\mathrm{kg}$ pour l'incidence des erreurs de mesure sur les variations de $\mathrm{Y}-\widehat{\mathrm{Y}}, 25,2 \mathrm{~kg}$ contre $2 \mathrm{I}, 8 \mathrm{~kg}$ pour l'incidence des erreurs d'ajustement proprement dites.

\section{CONCIUSION}

Ce travail n'avait pas la prétention d'analyser dans le détail l'ensemble des problèmes posés par l'établissement de formules barymétriques chez les bovins : son seul but était de rassembler les études que nous avons réalisées depuis ro ans en France à l'occasion de l'élaboration de ces formules pour divers organismes.

Une recherche complète sur ce point nécessiterait de connaitre la croissance relative du poids et des mesures corporelles utilisées ainsi que ses facteurs de variation sur des échantillons d'animaux moins disparates que ceux que nous possédions.

Il reste que les conclusions que l'on peut tirer de ce travail et des recherches déjà entreprises sont fonction non seulement des résultats obtenus mais aussi des possibilités pratiques.

En ce qui concerne le choix des mesures, si le tour spiral fournit en général d'aussi bonnes estimations que le tour de poitrine, l'utilisation combinée de ces 2 mesures au lieu du seul tour de poitrine est d'autant plus intéressante que les animaux sont plus âgés: la mesure du tour spiral pose néanmoins des difficultés pratiques (présence d'un deuxième opérateur) parfois insurmontables.

L'ajustement linéaire est satisfaisant pour les populations d'animaux adultes. Dans le cas des jeunes en croissance l'emploi des puissances des variables améliore nettement, comme l'ont montré la plupart des auteurs, la précision des ajustements. Toutefois, dans ce cas, l'établissement de plusieurs équations linéaires, par classes de poids ou d'âge fournit en général de meilleurs résultats. Là aussi un compromis 
devra être trouvé entre les inconvénients pratiques de la multiplication des formules qu'on peut traduire simplement, il est vrai, par cles abaques, et la recherche d'une précision maximum.

En ce qui concerne les autres facteurs de variation des ajustements, nous avons souligné l'incidence considérable de l'état musculaire des animaux dans un échantillon très hétérogène.

Quant à l'intérêt pratique de la barymétrie, nous avons montré qu'à l'échelon. de programmes de contrôle du poids en vue de la sélection sur descendance, il était plus rentable de l'utiliser si son cồt unitaire était inférieur de plus de Io p. Ioo à celui d'une pesée.

Reçu pour publication en novembre 1965.

\section{REMERCIEMENTS}

Nous tenons ì remercier ici les techniciens de l'I.X.R.A. et les nombreux organismes de vulgarisation administratifs ou professionnels, nationaux ou locaux, qui ont collaboré à la collecte de l'information sur laquelle porte ce travail. Nous remercions également MM. POLY et MÉRAT des conseils qu'ils nous ont donnés pour la rédaction de ce mémoire.

\section{SUMMARY}

ESTIMATION OF LIVE WEIGH'T IN CATTLE.

AN INVESTIGATION ON ITS POSSIBLE USES

Animals of different breeds, sexes, ages and fatness have been tested in France for ten years in order to find out formulas of live weight estimation by measurements on the account of various. breeders associations.

After having shown up the interest of chest and spiral girth in prediction of live weight, the respective reliabilities of these two measurements were compared and it was found that chest girth measurement had greater efficiency in growing cattle.

The accuracy of the various mathematical adjustments was calculated. We more particularly considered linear adjustments taking into account the square of chest girth and cube of spiral girth : the advantage of these adjustments in comparison with the linear adjustment of weight on chest and spiral girth is evident in growing animals. Age, fat ness and sex strongly influence the values of regression coefficients of body measurements on weight.

The standard error of prediction calculated from the best adjustments was approximately $4 \mathrm{p}$. Ioo of them. $1 / 4$ of the variance of errors is due to a lack precision when weighing and measuring, 3:4 to. actual errors of adjustments. When predicting the average weight of animals in the case of progeny test, a precision equivalent to that given by weighing can be obtained by measuring ten per cent more animals from each bull's progeny.

\section{RÉFÉRENCES BIBI,IOGRAPHIQUES}

Auriol P., Luplax J. M., 1960 . Recherches barymétriques sur les veaux de race Charollaise. C.R. Acad. Agric., 46, 938-943.

Bagot F. I., 1956. The relation between body dimensions and body weight in Sinhala cattle. Anim. Breed. Absir., 24, 127.

BERGE S., 1953. Chest circumference, live weight and slaughter weight in Norvegian breeds of cattle. Anim. Breed. Abstr., 21, 130. 
Branton C., Salisbury G. W., 1946. The estimation of the weight of bulls from heart girth measurements. J. Dairy Sci., 29, I41-1 43 .

Brody S., 1945. Bioenergelics and growlh. Reinhold Publishing Corporation, New York. 1023 pp.

Brookes A. J., Harringtov G., ig6o. Studies in beef production II. The estimation of live weight of beef steers from chest girth and other body measurements. J. agric. Sci., 55, 207-213.

BURT A. W. A., 1957. The comprarative efficiency of some methods of estimating the live weight of dairy cows. Anim. Breed. Abslr., 25, 357.

Davis H. P., Horgan R. H., Brovy S., Ragsdale A. C., r937. Relation of height at withers and chest girth to live weight of dairy cattle of different breeds and ages. Res. Bull. Neb. Agric. Fxper. Sia., 91, 29 pp.

Delage J., POLy J., Vissac B., 1955. Jitude de l'efficacité relative des diverses formules de barymétrie applicables aux bovins. Ann. Zootech., 4, $219^{-2} 31$.

Hansson A., 1926. Försök med Kreatursviktmattbandet Arax sommaren 1925. Sienska Betes-o Vallfröen. Arsskr., 8, I $48-160$.

Hvidstex H., I940. Beregning av vekta hos storfe ettev mal. 49 Bereln. Foringsforsok. Vorg. Landbrhogsk., $39 \mathrm{Pp}$.

Johansson I., IILLeman S. E., i 954. 'The relationship between certain body measurements and live and slaughter weight in cattle. Atim. Breed. Abstr., 22, 1-17.

Johanssox I., Vexise O., 195I. Studies on the values of various morphological characters for the diagnostic of monozygocity of cattle twins. Z. Tierz. ZiichBiol., 59, 389-424.

Le Rouldey M., Vissac B.. Poty J., Cinntet I'., 195\%. Une formule de barymétrie utilisable chez les femelles de race Normande dans des conditions normales d'exploitation. C. R. Acad. Agric., 44, 508$5 \mathrm{I} 3$.

Lusil J. L., Corbland O. C., 1930. A study of the accuracy of measurements of dairy cattle. J. agric. Res., 41, 37-49.

Marce J., LamuYe J., Cordez E., i953. 1 : Connaissances du bétail. Librairie agricole de la Maison Rusticulue, Paris, 208 pp.

Mougin P., Auriol P., 1960. Établissement de formules barymétriques pour les vaches de race Montbéliarde. C. R. Acal. Agric., 46, $809-875$.

Neuvy A., 1962. Méthodes d'appréciation du poids vif des bovins par barymétric. Publication Union nationale des Liores généalogiques, 16 , rue Claude Bernard. I'aris $5^{\mathrm{e}}$.

OSTERgaARd P. A., 1950. Undersogelser vedrorende Jerseykvaegets vaegt, mal og ydelse $25 \mathrm{I}$, Berein. Forsgslab. Kbh., 47 pp.

Ragsdale A. C., Brody S., i935. Estimating live weights of dairy cattle. Unir. Missouri. Coll. Agric. agric. exper. Sla. Bull., 354.

Ross J. G., 1958. A method of estimating live weights in small Shorthorn zebu cattle from linear body measurements. Anim. Breed. Abstr., 26, $35^{2}$.

Stagsvoub P., 1949. Untregning av levendevekten hos Raukollokser pa grummlag av brystormfanget. Nord. Vel. Wed., 1, 564-569.

Stennsberc; V., 1925, Om Bestemmelse of Hornkvaegs Vacgt ved Hjealp of Maalingen. Nord. Jorlbr. torksen., 289-304.

STEensbikg V., OstergaARd P., I945. Forholdet mellen Brystomfäng og valegt 216 . Beretn Forsogslab. $K b h, 67-89$.

Szczekin - Krotow, I96r. Contribution to the estimation of live weight in cattle in relation to constitutional type. Anim. Breed. Abstr., 30, 167.

VisSAC B., 1959. Rapport sur des recherches françaises intéressant le testage des taurtaux sur les aptitudes à la production de viande de leurs descendants. Publicalion F. E. Z., 72-125. 